\author{
$n^{\circ}$ 2013-05 \\ Non-tradable S\&P 500 Index \\ And the Pricing of Its Traded \\ Derivatives \\ C. GOURIÉROUX ${ }^{1}$ \\ J. JASIAK ${ }^{2}$ \\ P. $X U^{3}$
}

February 2013

Les documents de travail ne reflètent pas la position du CREST et n'engagent que leurs auteurs.

Working papers do not reflect the position of CREST but only the views of the authors.

\footnotetext{
1 CREST and University of Toronto, Canada.

2 York University, Canada.

3 ESSEC Business School.
} 


\title{
Non-tradable S\&P 500 Index and the Pricing of Its Traded Derivatives
}

\author{
Christian Gourieroux, Joann Jasiak, and Peng $\mathrm{Xu}^{\ddagger}$
}

January 17, 2013

\begin{abstract}
We derive a coherent multi-factor model to price various derivatives such as forwards, futures and European options written on a same underlying asset which is potentially non-tradable. We consider both cases when the underlying asset is self-financed and tradable and when it is not, and show the difference between both cases. When the underlying asset is self-financed and tradable, an additional arbitrage condition has to be introduced and implies nontrivial parameter restrictions. These restrictions can be tested in practice to check if the derivatives are priced as if the underlying asset were self-financed and tradable.
\end{abstract}

Keywords: Index Derivatives, Non-tradable Index, Generalized Method of Moments, Mispricing

${ }^{*}$ CREST and University of Toronto.

${ }^{\dagger}$ York University.

${ }^{\ddagger}$ ESSEC Business School. 


\section{Introduction}

As argued in Xu (2006), the S\&P 500 Index is an artificial number constructed to reflect the evolution of the market. It is not a self-financed or a tradable portfolio and it cannot be replaced by a mimicking portfolio such as the SPDR due to the particular way the S\&P 500 Index is calculated, maintained, and the way dividends are (not) accounted for. The non-tradability of the S\&P 500 Index has significant implications on risk hedging and pricing constraints. For example, the well-known Black-Scholes model [See Black and Scholes (1973) and Merton (1973)] assumes that the underlying asset is tradable and follows a geometric Brownian Motion process with constant volatility. Therefore, the market is completed by the underlying asset itself and the risk involved can be fully hedged by the underlying asset. By the No-Arbitrage condition, the market price of risk is determined uniquely by the price of the underlying asset. All derivatives written on the underlying asset can be evaluated uniquely with this market price of risk, combined with the terminal condition of the respective derivatives. If the underlying asset is nontradable, the underlying asset cannot be used as part of the arbitrage strategy and the value of the underlying asset does not need to satisfy the No-Arbitrage condition. The risk associated with the underlying asset is not hedged by itself and the expected return of the underlying asset under the risk-neutral probability is not necessarily equal to the risk-free rate. Knowing only the value of the underlying asset, we do not know the price of the risk. Therefore the prices of options written on a non-traded underlying asset whose price follows a geometric Brownian Motion process do not have to be evaluated by the Black-Scholes formula. Similar ideas apply to many other models. For instance, the stochastic volatility models in Heston (1993) and Ball and Roma (1994) assume that the expected return of the underlying asset is equal to the risk-free rate under the riskneutral probability, i.e., that the underlying asset is tradable and the risk associated with the underlying asset is hedged by itself. In general, because of the non-tradability of the S\&P 500 Index, the prices of its options do not have to satisfy the restrictions imposed by the pricing models that are based on the assumption that the underlying asset is a 
security traded in the market.

In this paper, we introduce a coherent multi-factor model for pricing various derivatives such as forwards, futures and European options written on the non-tradable S\&P 500 Index, and derivatives written on the S\&P 500 futures. The model illustrates the relationship between the index and its futures, and the relationship between the index and its put and call options, when the underlying asset is non-tradable. We also consider what the prices of the derivatives should be, if the index were self-financed and tradable. The model explains why the prices of derivatives written on a tradable asset and a non-tradable asset can be different. Moreover, the model provides a framework to test whether the S\&P 500 derivatives are priced by the investors as if the index were self-financed and tradable. To test this hypothesis, we consider three nested models: the one-, two-, and three-factor model. Since the underlying factors are unobservable, we transform the pricing model into a state space model. The models are estimated by combining the spot, futures and options data and using the unscented Kalman Filter (UKF) method. The Wald tests strongly reject the null hypothesis that the derivatives are priced as if the index were self-financed and tradable in all three models. Our diagnostic analysis also shows that the multi-factor models are superior to the one-factor model. The model in this paper can be easily extended to price derivatives written on other non-tradable indices such as a retail price index, a meteorological index, an index summarizing the results of a set of insurance companies, or a population mortality index.

The rest of the paper is organized as follows: In Section 2, we present a coherent pricing model for pricing derivatives written on the S\&P 500. The Spot-Futures Parity and Put-Call Parity are also derived for the case of a non-tradable underlying index. In Section 3, we derive the parameter restrictions which characterize the derivative pricing if the index were tradable. This allows us to discuss how derivative prices can differ for tradable and non-tradable underlying assets. In Section 4, we discuss the estimation method and testing procedure. In Section 5, we report the empirical results. We conclude in Section 6. Technical results and details are gathered in the Appendices. 


\section{The Pricing Model}

Under the absence of arbitrage opportunity (AAO), the market prices have to be compatible with a valuation system based on stochastic discounting [Harrison and Kreps (1979)]. The pricing formulas can be written either in discrete time or in continuous time, according to the assumptions of discrete or continuous trading (and information sets). The modern pricing methodology requires a joint coherent specification of these historical and risk-neutral distributions. For this purpose, we follow the practice initially introduced by Constantinides (1992), which specifies a parametric historical distribution and a parametric stochastic discount factor.

\subsection{Assumptions}

\subsubsection{Historical Dynamics of the Index}

The value of the index at date $\mathrm{t}$ is denoted by $I_{t}$. We assume that the log-index satisfies a diffusion equation with affine drift and volatility functions of $\mathrm{K}$ underlying factors $\left\{x_{k, t}\right\}$, $k=1, \cdots, K$ :

\section{Assumption 1.}

$$
d \log I_{t}=\left(\mu_{0}+\sum_{k=1}^{K} \mu_{k} x_{k, t}\right) d t+\left(\gamma_{0}+\sum_{k=1}^{K} \gamma_{k} x_{k, t}\right)^{1 / 2} d w_{t}
$$

where $\left\{\mu_{k}\right\}$ and $\left\{\gamma_{k}\right\}, k=0, \cdots, K$ are constants, and $\left\{w_{t}\right\}$ is a Brownian motion.

The underlying factors summarize the dynamic features of the index. As seen in Equation (2.2), they are assumed to be independent Cox, Ingersoll and Ross (CIR) processes, independent of the standard Brownian motion $\left\{w_{t}\right\}$. Since the CIR processes are nonnegative, the volatility of the log-index is positive whenever parameters $\left\{\gamma_{k}\right\}, k=$ $0, \cdots, K$, are positive. This positive parameter restriction is imposed in the rest of the paper. 
Assumption 2. The CIR processes $\left\{x_{k, t}\right\}, k=1, \cdots, K$ satisfy the stochastic differential equations:

$$
d x_{k, t}=\xi_{k}\left(\zeta_{k}-x_{k, t}\right) d t+\nu_{k} \sqrt{x_{k, t}} d w_{k, t}, \quad k=1, \cdots, K
$$

where $\xi_{k}, \zeta_{k}$ and $\nu_{k}$ are positive constants and $\left\{w_{k, t}\right\}, k=1, \cdots, K$ are standard independent Brownian motions, independent of $\left\{w_{t}\right\}$.

The condition $\xi_{k} \zeta_{k}>0$ ensures the nonnegativity of the CIR process (for a positive initial value $x_{0}>0$ ), while the conditions $\xi_{k}>0$ and $\zeta_{k}>0$ imply the stationarity of the CIR process. The condition $\nu_{k}>0$ can always be assumed for identifiability reason.

This general specification of the index dynamics includes the Black-Scholes model [Black and Scholes (1973)], when $\mu_{k}=\gamma_{k}=0, k=1, \cdots, K$, the stochastic volatility model considered by Heston (1993) and Ball and Roma (1994), when $K=1$ and $x_{1}$ is interpreted as a stochastic volatility, or the model with stochastic dividend yield [see Schwartz (1997) for example], when $K=1$ and $x_{1}$ appears in the drift only.

The transition distribution of the integrated CIR process is required for derivative pricing. This distribution is characterized by the conditional Laplace transform $E_{t}\left[\exp \left(-z \int_{t}^{t+h} x_{k, \tau} d \tau\right)\right]$, where $E_{t}$ denotes the conditional expectation given the past values of the process and $\mathrm{z}$ is a nonnegative constant (or more generally a complex number), which belongs to the domain of the existence of the conditional Laplace transform. This domain does not depend on past factor realizations, that is, on the information set. The conditional Laplace transform admits a closed form expression [see e.g. Cox, Ingersoll and Ross (1985b)]. The conditional Laplace transform of the integrated CIR process is an exponential affine function of the current factor value. It is given by

$$
E_{t}\left[\exp \left(-z \int_{t}^{t+h} x_{k, \tau} d \tau\right)\right]=\exp \left[-H_{1}^{k}(h, z) x_{k, t}-H_{2}^{k}(h, z)\right],
$$


where

$$
\begin{aligned}
H_{1}^{k}(h, z)= & \frac{2 z\left(\exp \left[\varepsilon_{k}(z) h\right]-1\right)}{\left(\varepsilon_{k}(z)+\xi_{k}\right)\left(\exp \left[\varepsilon_{k}(z) h\right]-1\right)+2 \varepsilon_{k}(z)} \\
H_{2}^{k}(h, z)= & \frac{-2 \xi_{k} \zeta_{k}}{\nu_{k}^{2}}\left\{\log \left[2 \varepsilon_{k}(z)\right]+\frac{h}{2}\left[\varepsilon_{k}(z)+\xi_{k}\right]\right. \\
& \left.-\log \left[\left(\varepsilon_{k}(z)+\xi_{k}\right)\left(\exp \left(\varepsilon_{k}(z) h\right)-1\right)+2 \varepsilon_{k}(z)\right]\right\} \\
\varepsilon_{k}(z)= & \sqrt{\xi_{k}^{2}+2 z \nu_{k}^{2}} .
\end{aligned}
$$

This formula also holds for a complex number $z=u+i v$, whenever $u>-1$ and $v \in \mathbf{R}$.

The joint dynamics of factors and log-index can be represented by means of the stochastic differential system:

$$
\begin{aligned}
& d\left[\begin{array}{c}
x_{1, t} \\
\vdots \\
x_{K, t} \\
\log I_{t}
\end{array}\right]=\left[\begin{array}{c}
\xi_{1}\left(\zeta_{1}-x_{1, t}\right) \\
\vdots \\
\xi_{K}\left(\zeta_{K}-x_{K, t}\right) \\
\mu_{0}+\sum_{k=1}^{K} \mu_{k} x_{k, t}
\end{array}\right] d t \\
&+\left[\begin{array}{cccc}
\nu_{1} \sqrt{x_{1, t}} & 0 & \cdots & 0 \\
0 & \ddots & & \vdots \\
\vdots & & \nu_{K} \sqrt{x_{K, t}} & 0 \\
0 & \cdots & 0 & \left(\gamma_{0}+\sum_{k=1}^{K} \gamma_{k} x_{k, t}\right)^{1 / 2}
\end{array}\right]
\end{aligned}
$$

where both the drift vector and the volatility-covolatility matrix are affine functions of the current values of the joint process $\left(x_{1, t}, \cdots, x_{K, t}, \log I_{t}\right)^{\prime}$. Thus, the stacked process $\left(x_{1, t}, \cdots, x_{K, t}, \log I_{t}\right)^{\prime}$ is an affine process [see Duffie and Kan (1996)], and the conditional Laplace transform of the integrated process $E_{t}\left[\exp \int_{t}^{t+h}\left(z_{1} x_{1, \tau}+\cdots+z_{K} x_{K, \tau}+z \log I_{\tau}\right) d \tau\right]$ will also admit an exponential affine closed form expression.

\subsubsection{Specification of the Stochastic Discount Factor}

The model is completed by a specification of a stochastic discount factor (SDF), which is used later on to price all derivatives written on the index. 
Assumption 3. The stochastic discount factor (SDF) for period $(t, t+d t)$ is

$$
\begin{aligned}
M_{t, t+d t} & =\exp \left(d m_{t}\right)=\exp \left[\left(\alpha_{0}+\sum_{k=1}^{K} \alpha_{k} x_{k, t}\right) d t+\beta d \log I_{t}\right] \\
& =\exp \left\{\left[\alpha_{0}+\beta \mu_{0}+\sum_{k=1}^{K}\left(\alpha_{k}+\beta \mu_{k}\right) x_{k, t}\right] d t+\beta\left(\gamma_{0}+\sum_{k=1}^{K} \gamma_{k} x_{k, t}\right)^{1 / 2} d w_{t}\right\} .
\end{aligned}
$$

This SDF explains how to correct for risk when pricing derivatives. The "risk premia" depend on the factors and index values, whereas the sensitivities of this correction with respect to these risk variables are represented by the $\alpha$ and $\beta$ parameters. The market price of risk associated with $w_{t}$ is ${ }^{1}-\beta\left(\gamma_{0}+\sum_{k=1}^{K} \gamma_{k} x_{k, t}\right)^{1 / 2}$. This specification of the SDF implicitly assumes that the market prices of the risk factors $\left\{w_{k, t}\right\}, k=1, \cdots, K$ are 0. Equivalently, Equation (2.2) also describes the risk-neutral distribution of $\left\{x_{k, t}\right\}$, $k=1, \cdots, K$. Under the risk-neutral probability, the joint dynamics of the underlying factors and log-index can be represented by means of the stochastic differential system:

$$
\begin{aligned}
d\left[\begin{array}{c}
x_{1, t} \\
\vdots \\
x_{K, t} \\
\log I_{t}
\end{array}\right]=\left[\begin{array}{c}
\xi_{1}\left(\zeta_{1}-x_{1, t}\right) \\
\vdots \\
\xi_{K}\left(\zeta_{K}-x_{K, t}\right) \\
\left.\mu_{0}+\beta \gamma_{0}+\sum\left(\mu_{k}+\beta \gamma_{k}\right) x_{k, t}\right)
\end{array}\right] d t \\
+\left[\begin{array}{cccc}
\nu_{1} \sqrt{x_{1, t}} & 0 & \ldots & 0 \\
0 & \ddots & & \vdots \\
\vdots & & \nu_{K} \sqrt{x_{K, t}} & 0 \\
0 & \ldots & 0 & \sqrt{\gamma_{0}+\sum \gamma_{k} x_{k, t}}
\end{array}\right]\left[\begin{array}{c}
d w_{1, t} \\
\vdots \\
d w_{K, t} \\
d w_{t}^{*}
\end{array}\right],
\end{aligned}
$$

where $\left\{w_{k, t}\right\}, k=1, \cdots, K$, and $\left\{w_{t}^{*}\right\}$ are standard independent Brownian motions under the risk-neutral probability. Thus only the last row is corrected for risk. This differential stochastic system is still an affine process.

\footnotetext{
${ }^{1}$ This can be seen easily from the short rate computed in Equation (2.14).
} 


\subsection{Pricing Formulas for European Derivatives Written on the Index}

As mentioned above, the arbitrage pricing proposes a valuation approach, which is compatible with observed market prices and proposes coherent quotes for non-highlytraded derivatives. More precisely, the value (price) at t of a European derivative paying $g\left(x_{1, t+h}, \cdots, x_{K, t+h}, I_{t+h}\right)$ at time $t+h$ is

$$
c(t, t+h, g)=E_{t}\left[\exp \left(\int_{t}^{t+h} d m_{\tau}\right) g\left(x_{1, t+h}, \cdots, x_{K, t+h}, I_{t+h}\right)\right] .
$$

The valuation formula is not assumed to be unique. Indeed, the SDF has been

parameterized by $\alpha_{0}, \alpha_{1}, \cdots, \alpha_{K}, \beta$, but the parameter values have not been fixed exante. Thus, we propose implicitly different possible valuations and by comparing with observed derivative prices, we estimate ex-post which one(s) is(are) compatible with observed market prices.

The aim of this section is to derive explicit valuation formulas for European index derivatives. All the formulas are derived from the valuation of European Index derivatives with power payoff. Such derivatives are not traded or more generally quoted. But these basic computations are used to derive:

- the risk-free term structure of interest rates

- the forward and futures prices of the index

- the prices of European options written on the index.

\subsubsection{Power Derivatives Written on the Index}

The following proposition is proved in Appendix A. 
Proposition 1. The value at $t$ of the European derivative paying $\exp \left[u \log \left(I_{t+h}\right)\right]=$ $\left(I_{t+h}\right)^{u}$ at maturity $t+h$ is

$$
\begin{aligned}
C(t, t+h, u) & =E_{t}\left[\left(I_{t+h}\right)^{u} \exp \left(\int_{t}^{t+h} d m_{\tau}\right)\right] \\
& =E_{t}\left[\exp \left(\int_{t}^{t+h} d m_{\tau}+u \log I_{t+h}\right)\right] \\
& =\exp \left(u \log I_{t}\right) \exp \left[-h z_{0}(u)-\sum_{k=1}^{K} H_{1}^{k}\left(h, z_{k}(u)\right) x_{k, t}-\sum_{k=1}^{K} H_{2}^{k}\left(h, z_{k}(u)\right)\right],
\end{aligned}
$$

where

$$
\begin{aligned}
z_{k}(u) & =-\alpha_{k}-(\beta+u) \mu_{k}-\frac{\gamma_{k}}{2}(\beta+u)^{2}, \quad \forall k=0, \cdots, K, \\
& =z_{k}(0)+u l_{k}+\frac{\gamma_{k}}{2} u(1-u), \\
l_{k} & =-\mu_{k}-\frac{1+2 \beta}{2} \gamma_{k}, \quad \forall k=0, \cdots, K,
\end{aligned}
$$

and $H_{1}^{k}(\cdot, \cdot)$ and $H_{2}^{k}(\cdot, \cdot)$ are given in equation(2.4).

Proposition 1 holds, if and only, if $z_{k}(u)>-1, \forall k=1, \cdots, K$. When we apply this formula to different traded derivatives, i.e., different values of $\mathrm{u}$, the inequalities above imply restrictions on parameters $\alpha, \beta$ and $\gamma$.

\subsubsection{The Risk-free Term Structure}

The zero-coupon bonds correspond to a unitary payoff, and their prices $B(t, t+h)$ correspond to the special case of $C(t, t+h, u)$ where $u=0$. The continuously compounded risk-free interest rates are defined by $r(t, t+h)=-\frac{1}{h} \log B(t, t+h)$. We get the following proposition:

Proposition 2. The prices of the zero-coupon bonds are:

$$
\begin{aligned}
B(t, t+h) & =C(t, t+h, 0) \\
& =\exp \left[-h z_{0}(0)-\sum_{k=1}^{K} H_{1}^{k}\left(h, z_{k}(0)\right) x_{k, t}-\sum_{k=1}^{K} H_{2}^{k}\left(h, z_{k}(0)\right)\right]
\end{aligned}
$$


where $z_{k}(\cdot)$ is defined in Equation (2.8), and $H_{1}^{k}(\cdot, \cdot)$ and $H_{2}^{k}(\cdot, \cdot)$ are given in equation(2.4). We deduce the expressions of the interest rates:

$$
\begin{aligned}
r(t, t+h) & =-\frac{1}{h} \log B(t, t+h) \\
& =-\frac{1}{h}\left[-h z_{0}(0)-\sum_{k=1}^{K} H_{1}^{k}\left(h, z_{k}(0)\right) x_{k, t}-\sum_{k=1}^{K} H_{2}^{k}\left(h, z_{k}(0)\right)\right] \\
& =z_{0}(0)+\frac{1}{h} \sum_{k=1}^{K} H_{1}^{k}\left(h, z_{k}(0)\right) x_{k, t}+\frac{1}{h} \sum_{k=1}^{K} H_{2}^{k}\left(h, z_{k}(0)\right) .
\end{aligned}
$$

The risk-free interest rates are affine functions of the CIR risk factors. This specification is the standard affine term structure model introduced in Duffie and Kan (1996) [see also Dai, Singleton (2000)]. It includes the one-factor CIR model [Cox, Ingersoll and Ross (1985b)] as well as the multi-factor term structure model of Chen and Scott (1993).

As explained in subsection 2.2.1, the following restrictions are imposed on the parameters:

$$
z_{k}(0)=-\alpha_{k}-\beta \mu_{k}-\frac{\gamma_{k}}{2} \beta^{2}>-1, \quad \forall k=1, \cdots, K .
$$

The short rate is defined by $r(t)=\lim _{h \rightarrow 0}-\frac{1}{h} \log B(t, t+h)$. The following proposition is proved in Appendix B.

Proposition 3. The short rate is given by

$$
\begin{aligned}
r(t) & =\lim _{h \rightarrow 0}-\frac{1}{h} \log B(t, t+h)=\left.\frac{d[-\log B(t, t+h)]}{d h}\right|_{h=0} \\
& =z_{0}(0)+\sum_{k=1}^{K} z_{k}(0) x_{k, t} .
\end{aligned}
$$

\subsubsection{Forward Prices for the S\&P 500 Index}

A forward contract is an agreement to deliver or receive a specified amount of the underlying asset (or equivalent cash value) at a specified price and date. A forward contract always has zero value when it is initiated. There is no money exchange initially or during the life of the contract, except at the maturity date when the price paid is equal to the specified forward price. The following proposition is proved in Appendix C. 
Proposition 4. The forward prices are given by

$$
\begin{aligned}
f(t, t+h)= & \frac{C(t, t+h, 1)}{C(t, t+h, 0)} \\
= & I_{t} \exp \left\{-h l_{0}-\sum_{k=1}^{K} H_{1}^{k}\left(h, z_{k}(1)\right) x_{k, t}+\sum_{k=1}^{K} H_{1}^{k}\left(h, z_{k}(0)\right) x_{k, t}\right. \\
& \left.-\sum_{k=1}^{K} H_{2}^{k}\left(h, z_{k}(1)\right)+\sum_{k=1}^{K} H_{2}^{k}\left(h, z_{k}(0)\right)\right\},
\end{aligned}
$$

where $z_{k}(\cdot)$ is defined in Equation (2.8), $l_{0}$ is defined in Equation (2.10), and $H_{1}^{k}(\cdot, \cdot)$ and $H_{2}^{k}(\cdot, \cdot)$ are given in equation(2.4).

In addition to the restrictions in (2.13), the following restrictions are imposed on the parameters:

$$
z_{k}(1)=-\alpha_{k}-(\beta+1) \mu_{k}-\frac{\gamma_{k}}{2}(\beta+1)^{2}=z_{k}(0)+l_{k}>-1, \quad \forall k=1, \cdots, K .
$$

\subsubsection{Futures Prices}

Let us now consider the price at $\mathrm{t}$ of a futures contract written on $I_{t+h}$. The major difference between a futures contract and a forward contract is the mark-to-market practice for the futures. A futures contract has also zero value when it is issued and there is no money exchange initially. However, at the end of each trading day during the life of the contract, the party against whose favor the price changes must pay the amount of change to the winning party. That is, a futures contract always has zero value at the end of each trading day during the life of the contract. If the interest rate is stochastic, the forward price and futures price are generally not the same [See Cox, Ingersoll and Ross (1981) and French (1983)]. The following proposition is proved in Appendix D.

Proposition 5. The prices at $t$ of futures written on $I_{t+h}$ are given by

$$
\begin{aligned}
F_{t, t+h} & =E_{t}\left[\exp \left(\int_{t}^{t+h} d m_{\tau}\right) \exp \left(\int_{t}^{t+h} r_{\tau} d \tau\right) I_{t+h}\right] \\
& =I_{t} \exp \left[-h l_{0}-\sum_{k=1}^{K} H_{1}^{k}\left(h, l_{k}\right) x_{k, t}-\sum_{k=1}^{K} H_{2}^{k}\left(h, l_{k}\right)\right],
\end{aligned}
$$

where $l_{k}$ is defined in Equation (2.10), and $H_{1}^{k}(\cdot, \cdot)$ and $H_{2}^{k}(\cdot, \cdot)$ are given in equation(2.4). 
As explained earlier, in addition to the restrictions in (2.13), the following restrictions are imposed on the parameters:

$$
l_{k}=-\mu_{k}-\frac{1+2 \beta}{2} \gamma_{k}>-1 \quad \forall k=1, \cdots, K .
$$

Propositions 4 and 5 show that

$$
f(t, t+h)=E_{t}\left[\exp \left(\int_{t}^{t+h} d m_{\tau}\right) \exp (r(t, t+h) h) I_{t+h}\right]
$$

and

$$
F_{t, t+h}=E_{t}\left[\exp \left(\int_{t}^{t+h} d m_{\tau}\right) \exp \left(\int_{t}^{t+h} r_{\tau} d \tau\right) I_{t+h}\right] .
$$

Since the short rate is stochastic, the forward and futures prices are not equal in general. A sufficient condition for the forward and futures prices to be identical is $z_{k}(0)=0$, $\forall k=1, \cdots, K$, i.e., the interest rates are non-stochastic. This is Proposition 3 in Cox, Ingersoll and Ross (1981).

\subsubsection{European Call and Put Options Written on the Index}

The prices of the European options are deduced by applying a transform analysis to function $C(t, t+h, u)$ computed for pure imaginary argument $\mathrm{u}$ [see Duffie, Pan and Singleton (2000) and Appendix E].

\section{Proposition 6.}

i) The European call prices are given by

$$
\begin{aligned}
G(t, t+h, X)= & E_{t}\left\{\exp \left(\int_{t}^{t+h} d m_{\tau}\right)\left[\exp \left(\log I_{t+h}\right)-X\right]^{+}\right\} \\
= & \frac{C(t, t+h, 1)}{2}-\frac{1}{\pi} \int_{0}^{\infty} \frac{\operatorname{Im}[C(t, t+h, 1-i v) \exp (i v \log X)]}{v} d v \\
& \quad-X\left\{\frac{C(t, t+h, 0)}{2}-\frac{1}{\pi} \int_{0}^{\infty} \frac{\operatorname{Im}[C(t, t+h,-i v) \exp (i v \log X)]}{v} d v\right\}
\end{aligned}
$$

where $X$ is the strike price, $h$ is the time-to-maturity, $i$ denotes the pure imaginary number and $\operatorname{Im}(\cdot)$ is the imaginary part of a complex number. 
ii) The European put prices are given by

$$
\begin{aligned}
H(t, t+h, X)= & E_{t}\left\{\exp \left(\int_{t}^{t+h} d m_{\tau}\right)\left[X-\exp \left(\log I_{t+h}\right)\right]^{+}\right\} \\
= & -\frac{C(t, t+h, 1)}{2}+\frac{1}{\pi} \int_{0}^{\infty} \frac{\operatorname{Im}[C(t, t+h, 1+i v) \exp (-i v \log X)]}{v} d v \\
& +X\left\{\frac{C(t, t+h, 0)}{2}-\frac{1}{\pi} \int_{0}^{\infty} \frac{\operatorname{Im}[C(t, t+h, i v) \exp (-i v \log X)]}{v} d v\right\} .
\end{aligned}
$$

Again, the restrictions (2.13) and (2.16) are imposed ${ }^{2}$.

In particular, the relationship between the prices of European call and put options is

$$
\begin{aligned}
G(t, t+h, X)-C(t, t+h, 1)=H( & , t+h, X)-X C(t, t+h, 0) \\
& -\frac{1}{\pi} \int_{0}^{\infty} \frac{\operatorname{Im}[C(t, t+h, 1-i v) \exp (i v \log X)]}{v} d v \\
& +\frac{1}{\pi} \int_{0}^{\infty} \frac{\operatorname{Im}[C(t, t+h, 1+i v) \exp (-i v \log X)]}{v} d v \\
& +\frac{X}{\pi} \int_{0}^{\infty} \frac{\operatorname{Im}[C(t, t+h,-i v) \exp (i v \log X)]}{v} d v \\
& -\frac{X}{\pi} \int_{0}^{\infty} \frac{\operatorname{Im}[C(t, t+h, i v) \exp (-i v \log X)]}{v} d v
\end{aligned}
$$

Equation (2.23) provides the deviation to the Put-Call Parity due to the non-tradability of the underlying index and shows that this deviation is stochastic.

\subsection{Pricing Formulas for European Derivatives Written on Fu- tures}

\subsubsection{Derivatives Written on Futures}

As for derivatives written on the index, we first consider European derivatives written on futures with exponential payoffs. More precisely, we introduce three different dates:

- $\mathrm{t}$ is the current date

${ }^{2} \operatorname{Re}\left(z_{k}(1-i v)\right)>-1, \operatorname{Re}\left(z_{k}(-i v)\right)>-1, \operatorname{Re}\left(z_{k}(1+i v)\right)>-1$ and $\operatorname{Re}\left(z_{k}(i v)\right)>-1, \forall k=1, \cdots, K$, where $\operatorname{Re}(\cdot)$ denotes the real part of a complex number, should also hold in order for the pricing formulas to exist. $\operatorname{Re}\left(z_{k}(1-i v)\right)=\operatorname{Re}\left(z_{k}(1+i v)\right)=-\alpha_{k}-(\beta+1) \mu_{k}-\frac{\gamma_{k}}{2}(\beta+1)^{2}+\frac{\gamma_{k}}{2} v^{2}=z_{k}(1)+\frac{\gamma_{k}}{2} v^{2}$ and $\operatorname{Re}\left(z_{k}(-i v)\right)=\operatorname{Re}\left(z_{k}(i v)\right)=-\alpha_{k}-\beta \mu_{k}-\frac{\gamma_{k}}{2} \beta^{2}+\frac{\gamma_{k}}{2} v^{2}=z_{k}(0)+\frac{\gamma_{k}}{2} v^{2}$. So the restrictions (2.13)and (2.16)are sufficient. 
- $\mathrm{t}+\mathrm{h}$ is the maturity date of the derivatives on futures

- $\mathrm{t}+\mathrm{h}+\mathrm{m}$ is the maturity date of the futures on which the derivatives are written.

$C^{F}(t, t+h, t+h+m, u)$ denotes the price at $t$ of the European derivative paying $\left(F_{t+h, t+h+m}\right)^{u}$ at $t+h$. The following proposition is proved in Appendix F.

Proposition 7. The prices at $t$ of the European derivatives paying $\left(F_{t+h, t+h+m}\right)^{u}$ at $t+h$ are given by

$$
\begin{aligned}
& C^{F}(t, t+h, t+h+m, u) \\
= & E_{t}\left[\exp \int_{t}^{t+h} d m_{\tau}\left(F_{t+h, t+h+m}\right)^{u}\right] \\
= & \exp \left(u \log I_{t}\right) \exp \left\{m\left(u \mu_{0}-\frac{1+2 \beta}{2} u \gamma_{0}\right)+h\left[(\beta+u) \mu_{0}+\frac{(\beta+u)^{2}}{2} \gamma_{0}+\alpha_{0}\right]\right. \\
& -\sum_{k=1}^{K} u H_{1}^{k}\left(m, l_{k}\right) h \xi_{k} \zeta_{k}-\sum_{k=1}^{K} u H_{2}^{k}\left(m, l_{k}\right)-\sum_{k=1}^{K} H_{2}^{k}\left(h, p_{k}(m, u)\right) \\
& \left.-\sum_{k=1}^{K}\left[u H_{1}^{k}\left(m, l_{k}\right)+H_{1}^{k}\left(h, p_{k}(m, u)\right)\right] x_{k, t}\right\}
\end{aligned}
$$

where

$p_{k}(m, u)=-\alpha_{k}-(\beta+u) \mu_{k}-u H_{1}^{k}\left(m, l_{k}\right) \xi_{k}-\frac{\gamma_{k}}{2}(\beta+u)^{2}-\frac{u^{2}}{2}\left[H_{1}^{k}\left(m, l_{k}\right)\right]^{2} \nu_{k}^{2} \quad \forall k=1, \cdots, K$,

$l_{k}$ is given in equation(2.10), and $H_{1}^{k}(\cdot, \cdot)$ and $H_{2}^{k}(\cdot, \cdot)$ are given in equation(2.4).

Again, we impose (2.13) and (2.18) as well as the following restrictions on the parameters:

$$
\begin{gathered}
p_{k}(m, u)=-\alpha_{k}-(\beta+u) \mu_{k}-u H_{1}^{k}\left(m, l_{k}\right) \xi_{k}-\frac{\gamma_{k}}{2}(\beta+u)^{2}-\frac{u^{2}}{2}\left[H_{1}^{k}\left(m, l_{k}\right)\right]^{2} \nu_{k}^{2}>-1, \\
\forall k=1, \cdots, K .
\end{gathered}
$$

\subsubsection{European Call Options Written on Futures}

The following proposition is proved in Appendix G. 
Proposition 8. The prices of European calls written on futures are given by

$$
\begin{aligned}
& G^{F}(t, t+h, t+h+m, X) \\
= & E_{t}\left\{\exp \left(\int_{t}^{t+h} d m_{\tau}\right)\left[\exp \left(\log F_{t+h, t+h+m}\right)-K\right]^{+}\right\} \\
=\frac{C^{F}(t, t+h, t+h+m, 1)}{2} & \\
& -\frac{1}{\pi} \int_{0}^{\infty} \frac{\operatorname{Im}\left[C^{F}(t, t+h, t+h+m, 1-i v) \exp (i v \log X)\right]}{v} d v \\
& -X\left\{\frac{C^{F}(t, t+h, t+h+m, 0)}{2}\right. \\
& \left.-\frac{1}{\pi} \int_{0}^{\infty} \frac{\operatorname{Im}\left[C^{F}(t, t+h, t+h+m,-i v) \exp (i v \log X)\right]}{v} d v\right\}
\end{aligned}
$$

The parameters are subject to the restrictions in (2.13), (2.18) and ${ }^{3}$

$$
\begin{gathered}
p_{k}(m, 1)=-\alpha_{k}-(\beta+1) \mu_{k}-H_{1}^{k}\left(m, l_{k}\right) \xi_{k}-\frac{\gamma_{k}}{2}(\beta+1)^{2}-\frac{1}{2}\left[H_{1}^{k}\left(m, l_{k}\right)\right]^{2} \nu_{k}^{2}>-1, \\
\forall k=1, \cdots, K .
\end{gathered}
$$

\section{Parameter Restrictions for a Tradable Index}

In Section 2, the pricing formulas are valid for tradable as well as non-tradable index. In this section, we derive the restrictions implied by the tradability of the underlying index.

When the benchmark index is a self-financed and tradable asset, the pricing formula is also valid for the index itself. In this case, we have an additional condition:

$$
I_{t}=E_{t}\left[\exp \left(\int_{t}^{t+h} d m_{\tau}\right) I_{t+h}\right]=C(t, t+h, 1)
$$

and

$$
C(t, t+h, 1)=I_{t} \exp \left[-h z_{0}(1)-\sum_{k=1}^{K} H_{1}^{k}\left(h, z_{k}(1)\right) x_{k, t}-\sum_{k=1}^{K} H_{2}^{k}\left(h, z_{k}(1)\right)\right],
$$

where $z_{k}(\cdot)$ is defined in Equation $(2.8)$, and $H_{1}^{k}(\cdot, \cdot)$ and $H_{2}^{k}(\cdot, \cdot)$ are given in equation (2.4). $z_{k}(1)>-1$ is imposed, $\forall k=1, \cdots, K$.

\footnotetext{
${ }^{3}$ Restrictions (2.13) imply $p_{k}(m, 0)=z_{k}(0)>-1$. The inequalities $p_{k}(m, 0)>-1$ and $p_{k}(m, 1)>-1$ imply $\operatorname{Re}\left(p_{k}(m,-i v)\right)>-1$ and $\operatorname{Re}\left(p_{k}(m, 1-i v)\right)>-1$.
} 
This additional pricing condition has to be satisfied in any environment. By considering the expression of $C(t, t+h, 1)$ and identifying the different terms in the decomposition, we see that the dynamic parameters are constrained by

$$
\begin{cases}H_{1}^{k}\left(h, z_{k}(1)\right)=0, & \forall k=1, \cdots, K, \quad \forall h, \\ -h z_{0}(1)-\sum_{k=1}^{K} H_{2}^{k}\left(h, z_{k}(1)\right)=0, & \forall h,\end{cases}
$$

or equivalently by the conditions shown in Proposition 9 (See the proof in Appendix H).

Proposition 9. When the benchmark index is a self-financed and tradable asset, the dynamic parameters are constrained by

$$
z_{k}(1)=\alpha_{k}+(\beta+1) \mu_{k}+\frac{\gamma_{k}}{2}(\beta+1)^{2}=z_{k}(0)+l_{k}=0, \quad \forall k=0, \cdots, K .
$$

These restrictions fix the parameters $\left\{\alpha_{k}\right\}, k=0, \cdots, K$ of the SDF as functions of the parameters of the index dynamics.

When the benchmark index is tradable, the risk-neutral dynamics of $\log I_{t}$ can also be written as

$$
d \log I_{t}=\left[r(t)-\frac{\gamma_{0}+\sum \gamma_{k} x_{k, t}}{2}\right] d t+\sqrt{\gamma_{0}+\sum \gamma_{k} x_{k, t}} d w_{t}^{*}
$$

or

$$
\frac{d I_{t}}{I_{t}}=r(t) d t+\sqrt{\gamma_{0}+\sum \gamma_{k} x_{k, t}} d w_{t}^{*} .
$$

In other word, conditional on the underlying factors, the risk $w_{t}$ can be hedged by the index and the short rate if the index is tradable.

If the benchmark index is tradable, the formulas of derivative prices can be simplified. In particular, the forward price derived in Proposition 4 simplifies to the standard formula:

$$
f(t, t+h)=\frac{I_{t}}{B(t, t+h)},
$$

and the Spot-Futures Parity will hold for the index and its forward price. 


\section{Estimation Method and Testing Procedure}

We want to check if the restriction $z_{k}(1)=0$, for all $k=0, \cdots, K$, derived in Proposition 9, are satisfied. If they are satisfied, the market prices the derivatives written on the index as if the index were self-financed and tradable. To test the restrictions, we need to get an estimator of $z_{k}(1)$ for all $k=0, \cdots, K$. This can be achieved by combining the spot, futures and options data ${ }^{4}$. The pricing model in Section 2 can be estimated using the unscented Kalman Filter (UKF) method in Wan and Van Der Merwe (2000).

Equation (2.3) shows that we cannot identify $\varsigma_{k}, \nu_{k}^{2}$ and $z$ separately. Only $\frac{\varsigma_{k}}{\nu_{k}^{2}}$ and $z \nu_{k}^{2}$ can be identified. Equation (2.7) further shows that we can only identify $\mu_{k} \nu_{k}^{2}, \gamma_{k} \nu_{k}^{2}$, $\alpha_{k} \nu_{k}^{2}$ from the pricing formulae. Therefore, follow Dai and Singleton (2000), we normalize the model by setting $\nu_{k}=1$ for $k=1, \cdots, K$.

As the underlying factors $\left\{x_{k, t}\right\}$ are not observable, we transform the model into a dynamic state-space form and estimate it with a filtering method.

The state equations and measurement equations are specified as follows. Equation (2.2) is discretized daily (1/252 years) to generate the state equation for all factors. We consider one, two and three factors respectively.

We use daily data from the spot index, two index futures and three index options to estimate the model. Therefore we have six measurement equations. Equation (2.1) is discretized daily to generate the first measurement equation for the spot index. The annualized $\log$ of futures spot ratio (ALFSR) is defined $\operatorname{as}^{5} \frac{1}{h} \log \frac{F_{t, t+h}}{I_{t}}$, where $F_{t, t+h}$ is given by Equation (2.17). The measurement equations for the futures are generated by adding an error term to the ALFSR. The call options price in Equation (2.19) is normalized by dividing the corresponding Black-Scholes vega ${ }^{6}$, we denote it option pricevega ratio (OPVR). The measurement equations for the options are generated by adding

\footnotetext{
${ }^{4}$ Note that only futures and options data correspond to tradeable assets.

${ }^{5}$ If the spot-futures parity, $F_{t, t+h}=I_{t} e^{(r-q) h}$, where $r$ is the annually continuously compounded riskfree interest rate and $q$ is the dividend yield, holds for the index and its futures, then ALFSR simply equals the riskfree interest rate minus the dividend yield.

${ }^{6}$ The Black-Scholes vega is the derivative of the Black-Scholes options price with respect to the volatility.
} 
an error term to the OPVR. All the error terms for the futures and options are assumed to be independently and normally distributed with mean 0 and variance $\sigma_{i}^{2}, i=1, \cdots, 5$. The parameters to be estimated are $\mu_{k}, \gamma_{k}, \alpha_{k}, k=0, \cdots, K, \xi_{k}, \zeta_{k}, k=1, \cdots, K, \beta$ and $\sigma_{i}^{2}, i=1, \cdots, 5$. We use $\theta$ to denote the vector of all the parameters. The total number of parameters is $5 K+9$, where $K$ is the number of latent factors.

The above state-space model is Gaussian, but nonlinear. We thus implement the unscented Kalman Filter (UKF) method in Wan and Van Der Merwe (2000), which linearizes the model and removes the requirements to explicitly calculate Jacobians or Hessians without sacrificing the accuracy. Since all the error terms are assumed to be normally distributed in the state space model, the loglikelihood function of the observed variables $y_{t}=\left[d \log I_{t}, A L F S R_{1}, A L F S R_{2}, O P V R_{1}, O P V R_{2}, O P V R_{3}\right]^{\prime}$ is given by

$$
\log L_{t}(\theta)=-\frac{6}{2} \ln 2 \pi-\frac{1}{2} \ln \left|P_{y_{t}}^{-}\right|-\frac{1}{2}\left(y_{t}-\hat{y}_{t}^{-}\right)^{\prime}\left(P_{y_{t}}^{-}\right)^{-1}\left(y_{t}-\hat{y}_{t}^{-}\right)
$$

where $\hat{y}_{t}^{-}$is the predicted value of $y_{t}$ based on earlier observations and $P_{y_{t}}^{-}$is the predicted covariance matrix. The maximum likelihood estimator is obtained as

$$
\hat{\theta}=\operatorname{argmax}_{\theta} \sum_{t=1}^{T} \log L_{t}(\theta)
$$

where $\mathrm{T}$ is number of days in the dataset. Under standard regularity conditions, these estimators are asymptotically normal.

The "sandwich" formula is used to estimate the covariance of the quasi-maximum likelihood estimator (see White (1982) and Gourieroux, Monfort and Trognon (1984)) to take account of the possibility that the model is misspecified:

$$
\hat{\Sigma}_{\hat{\theta}}=\frac{1}{T} I(\hat{\theta})^{-1} J(\hat{\theta}) I(\hat{\theta})^{-1}
$$

where

$$
I(\hat{\theta})=-\frac{1}{T} \sum_{t=1}^{T} \frac{\partial^{2} \log L_{t}(\hat{\theta})}{\partial \theta \partial \theta^{\prime}} \quad \text { and } \quad J(\hat{\theta})=\frac{1}{T} \sum_{t=1}^{T} \frac{\partial \log L_{t}(\hat{\theta})}{\partial \theta} \frac{\partial \log L_{t}(\hat{\theta})}{\partial \theta^{\prime}}
$$


We deduce that the estimated functions of the parameters characterizing the restrictions for index tradability

$$
\widehat{z_{k}}(1)=\widehat{\alpha}_{k}+(\widehat{\beta}+1) \widehat{\mu}_{k}+\frac{\widehat{\gamma}_{k}}{2}(\widehat{\beta}+1)^{2}, \quad \forall k=0, \cdots, K
$$

are also asymptotically normal, with an estimated variance-covariance matrix $\widehat{\Omega}$, which is computed by the $\delta$-method. More precisely,

$$
\widehat{\mathbf{\Omega}}=\widehat{\mathbf{J}} \cdot \widehat{\mathbf{V}} \cdot \widehat{\mathbf{J}}^{\prime}
$$

where the Jacobian matrix $\widehat{\mathbf{J}}$ with size $K+1$ by $3 K+4$ is given by

$$
\widehat{\mathbf{J}}=\left[\begin{array}{ccccccccccccc}
\widehat{\beta}+1 & 0 & \cdots & 0 & \frac{(\widehat{\beta}+1)^{2}}{2} & 0 & \cdots & 0 & 1 & 0 & \cdots & 0 & \widehat{\mu}_{0}+\widehat{\gamma}_{0}(\widehat{\beta}+1) \\
0 & \ddots & & \vdots & 0 & \ddots & & \vdots & 0 & \ddots & & \vdots & \widehat{\mu}_{1}+\widehat{\gamma}_{1}(\widehat{\beta}+1) \\
\vdots & & \ddots & 0 & \vdots & & \ddots & 0 & \vdots & & \ddots & 0 & \vdots \\
0 & \cdots & 0 & \widehat{\beta}+1 & 0 & \cdots & 0 & \frac{(\widehat{\beta}+1)^{2}}{2} & 0 & \cdots & 0 & 1 & \widehat{\mu}_{K}+\widehat{\gamma}_{K}(\widehat{\beta}+1)
\end{array}\right]
$$

and $\widehat{\mathbf{V}}$ denotes the variance-covariance matrix of estimator $\left[\widehat{\mu}_{0} \cdots \widehat{\mu}_{K}, \widehat{\gamma}_{0} \cdots \widehat{\gamma}_{K}, \widehat{\alpha}_{0} \cdots \widehat{\alpha}_{K}\right.$, $\widehat{\beta}]^{\prime} . \widehat{\mathbf{V}}$ is a submatrix of $\hat{\Sigma}_{\hat{\theta}}$.

A Wald test statistic of the null-hypothesis that the derivatives are priced as if the index were self-financed and tradable is:

$$
\xi_{\mathrm{w}}=\widehat{z}(1)^{\prime} \widehat{\Omega}^{-1} \widehat{z}(1)
$$

Under the null-hypothesis this statistic follows asymptotically a chi-square distribution with $K+1$ degree of freedom.

\section{Empirical Results}

In this section, we present the estimation and test results. Subsection 5.1 introduces the data. The model is estimated with the quasi-maximum likelihood estimation method discussed in Section 4. The estimation and testing results are presented in Subsection 5.2. We also analyze the model performance and compare the one-, two-, and three-factor models in this subsection. 


\subsection{Description of Data}

In order to identify all the parameters in the model, we combined in the estimation the data of S\&P 500 index, index futures and index options. They are obtained from Optionmetrics. The spot index and the futures are only used for estimation, while the options data are divided into two parts, one for estimation and the other for out-of-thesample test.

The dataset ranges from January 3, 2001 to December 29, 2006. There are totally 1506 days. The summary statistics is presented in Table 1 and the plots of all the in-sample observations are shown in Figure 1.

The index level is computed using the last transaction prices of its component stocks. As seen in the table and the plots, the average daily change of S\&P 500 Index is very small, while its standard deviation is relatively larger. The variable $d \log I_{t}$ is positively skewed and has a fatter tail than the normal distribution.

The S\&P 500 futures contracts traded in Chicago Mercantile Exchange (CME) are among the most actively traded financial derivatives in the world. On each day, there are eight futures contracts with different maturity dates. The maturity dates are the third Friday of the eight months in the following March quarterly cycle (March, June, September and December). The futures contracts are ranked by their maturities and we selected two futures for estimation each day. The first futures (Fu1) has the shortest maturity except that in the March cycle months and before the maturity date the futures with the second shortest maturity is used. The second futures (Fu2) expires a quarter later than Fu1. Fu1 has time-to-maturity ranging from 15 to 112 days, while the maturity of Fu2 ranges from 105 to 204 days. These two futures usually have the highest trading volumes with open interest ${ }^{7}$ greater than 1700 contracts. The futures prices are quoted in terms of index points, and the contract size is $\$ 250$ times CME S\&P 500 futures price. The prices vary considerably during our sample period. The ALFSR, which is equal to $\frac{1}{h} \log \frac{F_{t, t+h}}{I_{t}}$, was more stable as seen in Figure 1. The ALFSR for the first futures has a

\footnotetext{
${ }^{7}$ Open interest refers to the total number of long (short) positions outstanding in an derivatives contract.
} 
mean of 0.009 and standard deviation of 0.018 , and the second one has a mean of 0.011 and standard deviation of 0.015 .

The S\&P 500 Index Options traded on the Chicago Board Options Exchange (CBOE) are European options. They are among the most liquid exchange-traded options and are extensively used for testing the option pricing models. The exchange-traded S\&P 500 Index Options differ from over-the-counter options and have a deterministic issuing dates, maturity dates and strikes to enhance the liquidity. The expiration months are the three near-term months followed by three additional months from the March quarterly cycle, plus two additional months from June and December. The expiration date is the Saturday following the third Friday of the expiration month. The underlying asset is the index level multiplied by 100. Strike price intervals are 5 points and 25 points for long term contracts.

In this paper, the options data are filtered as follows. First, only call options are included. Second, to alleviate the liquidity concern, we only consider call options with open interest greater than 100, trading volume greater than 0, maturity between 7 days and 540 days, and moneyness ${ }^{8}$ between 0.85 and 1.06. Third, to mitigate the market microstructure problem, we eliminate options with best bid prices less than $3 / 8$ dollars. The filtered dataset contains 1506 days, 77,224 options in total and 51 observations per day on average. A similar filtering approach was used in Li (2012). On each day, we select three call options for estimation and the rest are used for out-of-the-sample test. We try to use a variety of options with distinct moneyness and time-to-maturity in the estimation. We categorize three sets of options. The first set has time-to-maturity less than 60 days and moneyness between 0.97 and 1.03. These at-the-money short time-tomaturity (ATM-SM) options are among the most liquid products in the market. Options with medium and long maturity are generally more liquid when they are out-of-themoney. Therefore, the second set of options (OTM-MM) has time-to-maturity between 60 to 180 days and moneyness less than 1, and the third set (OTM-LM) has time-to-

\footnotetext{
${ }^{8}$ The moneyness is defined as the underlying price divided by the strike price, i.e., I/X.
} 
maturity longer than 180 days and moneyness less than 1. For estimation purpose, we choose from each set the option with the highest trading volume each day.

Figure 1 shows that the price-vega ratio for the options are high when the underlying index is volatile and low when the index is relatively stable. Options used for out-ofsample test has a wide range of time-to-maturity and moneyness, as shown in Table 1. The time-to-maturity varies from 10 days to 540 days, while the moneyness ranges from 0.85 to 1.06 .

\subsection{Estimation Results and Model Comparison}

Table 2 summarizes the estimation and testing results for the one-, two- and three-factor models. The table reports the parameter estimates and the standard deviations. The maximized loglikelihood for each model and the test statistics $\xi_{\mathbf{w}}$ for the null hypothesis of tradable index computed in equation (4.5) are presented at the end of the table.

In Table 2, we see that the standard errors of the parameter estimate in the one-factor model is very large. As a result, none of the estimates for the parameters in $\theta$ appears to be statistically significant. The Wald test statistic, $\xi_{\mathrm{w}}$, is equal to 846 . So the nullhypothesis that the derivatives are priced as if the index were self-financed and tradable is strongly rejected in the one-factor model.

Similarly, the estimates for most of the parameters in the two-factor model are not statistically significant. The Wald test statistic, $\xi_{\mathrm{w}}$, is equal to 997 . So the null-hypothesis that the derivatives are priced as if the index were self-financed and tradable is also strongly rejected in the two-factor model.

For the three-factor model, the estimates for most parameters become statistically significant, which means that a third CIR factor process is required. The estimate of 2.23, 2.23 and 1.72 for $\xi_{1}, \xi_{2}$ and $\xi_{3}$ implies that the underlying factors are mean reverse and they are not very persistent. The market price of risk associated with $w_{t}$ is $-\beta\left(\gamma_{0}+\right.$ $\left.\sum_{k=1}^{K} \gamma_{k} x_{k, t}\right)^{1 / 2}$. All $\gamma$ estimates are positive and the estimate of -5.66 for $\beta$ implies positive market risk premium. The Wald test statistic, $\xi_{\mathbf{w}}$, is equal to 18,232. So again 
the null-hypothesis that the derivatives are priced as if the index were self-financed and tradable is strongly rejected in the three-factor model despite the rather small forecasting error on $I_{t}$. In other words, significant mispricing of options and futures would appear if the three-factor model were estimated with the parameters constrained by the tradable restrictions (3.3) as usually done in the literature.

The results above are also compatible with "Roll's critique". Roll (1977) argues that the market portfolio in the Capital Asset Pricing Model is unobservable. In practice, a market index, such as the S\&P 500 Index, often serves as a proxy for the market portfolio. Our results show that the S\&P 500 Index cannot be considered as the value of a self-financed traded portfolio, in particular, not the value of the market portfolio.

The likelihood-ratio test of the one-factor model against two-factor model using the estimated loglikelihood rejects the one-factor model. Similarly, the two-factor model is rejected against the three-factor model.

Table 3 reports the in-sample pricing (forecasting) errors for the three models. The pricing errors are measured as the absolute difference between model implied price and the observed price as a percentage of the observed price. For the index which is not tradable, the interpretation is in terms of forecasting error. For the index, we report on the log index value, while for the futures and options, the actual prices are examined. There are 1,506 days in the data. The daily absolute percentage pricing (forecasting) errors for each model are shown in Figure 2 to Figure 4.

The in-sample pricing (forecasting) errors for spot index and index futures in all three models are very small due to the linearity of the pricing functions. The pricing errors are in general larger for the options, although all the mean values are less than $10 \%$. Figure 2 to Figure 4 shows that the pricing errors tend to be smaller in earlier period of the sample, when the underlying index and the latent factors are more volatile.

The factors in the models capture the risks that affect the expected return, the volatility and the dividend of the underlying index. Figure 5 plots the estimate of the filtered factors in each model. These three factors play different roles. Table 3 shows that the first factor corresponds to the short term risk, but does not fit the long end of the deriva- 
tives very well. When additional factors are included, the medium term and long term risks are better accounted for. This is clear by checking the in-sample pricing error of the medium-term futures $(\mathrm{Fu} 2)$ and the options with medium and long maturities (OP2 and OP3). In Table 3, the mean absolute percentage pricing error for the medium maturity futures $(\mathrm{Fu} 2)$ and the medium maturity options (OP2) are much smaller in the two-factor models, while the three-factor model produces the smallest pricing error for the long maturity options (OP3). The superiority of the two- and three-factor model can also been seen from the parameter estimates $\sigma_{F u 2}, \sigma_{O P 2}$ and $\sigma_{O P 3}$, which measure the mispricing of the derivatives with the medium and long maturity. The smaller values of these parameters in the multi-factor models indicate that the multi-factor models are more capable of explaining the variations in the observed data.

Table 4 reports the out-of-the-sample option pricing errors for the three models. There are 1,506 days and 72,706 out-of-the-sample observations in total. The options are divided into 15 groups based on the maturity and moneyness. The mean of absolute percentage option pricing errors for each group is reported.

As seen in the table, all the three models do poorly in estimating the deep out-ofthe-money options, especially those with short term maturities. However, the models produce more accurate estimates for the other options and the pricing errors for these options are fairly small. The two- and three-factor models perform better than the onefactor model in forecasting the prices of the medium- and long-term derivatives. The three-factor model yields the smallest mean absolute percentage pricing errors for the options with long maturities.

\section{Conclusion}

In this paper we consider a coherent multi-factor affine model to price various derivatives such as forwards, futures and European options written on the non-tradable S\&P 500 Index, and derivatives written on the S\&P 500 futures. 
We consider both cases when the underlying index is self-financed and tradable and when it is not, and show the difference between the two pricing models. When the underlying asset is self-financed and tradable, an additional arbitrage condition has to be introduced and implies additional parameter restrictions. These restrictions can be tested in practice to check whether the derivatives are priced as if the underlying index were self-financed and tradable.

To test the restrictions, we consider three nested factor models. The models are estimated by combining the spot, futures and options data and using the unscented Kalman Filter (UKF) method. The Wald tests strongly reject the null-hypothesis that the derivatives are priced as if the index were self-financed and tradable in all three models. In other words, i) significant mispiricing of options and futures contracts would be observed if the factor models were estimated with parameters constrained by the index tradability restriction as usually done in the literature; ii) it is not possible to reproduce the index by means of a self-financed mimicking portfolio without significant errors; iii) the S\&P 500 Index cannot be considered as the value of the market portfolio.

The S\&P 500 Index is not the only non-tradable index on which various derivatives have been written. Our model can be easily extended to price derivatives written on other non-tradable indices such as a retail price index, a meteorological index, an index summarizing the results of a set of insurance companies, or a population mortality index. These other application are even more appealing since no liquid mimicking portfolio is generally proposed for these indices on the market. 


\section{Appendices: Proofs of Propositions}

\section{A Proof of Proposition 1}

The price of the call option is:

$$
\begin{aligned}
& C(t, t+h, u) \\
& =E_{t}\left[\exp \left(\int_{t}^{t+h} d m_{\tau}+u \log I_{t+h}\right)\right] \\
& =E_{t}\left\{\exp \left[\int_{t}^{t+h} d m_{\tau}+u\left(\log I_{t}+\int_{t}^{t+h} d \log I_{\tau}\right)\right]\right\} \\
& =\exp \left(u \log I_{t}\right) E_{t}\left[\exp \left(\int_{t}^{t+h}\left(d m_{\tau}+u d \log I_{\tau}\right)\right]\right. \\
& =\exp \left(u \log I_{t}\right) E_{t}\left\{\operatorname { e x p } \int _ { t } ^ { t + h } \left(\left[\alpha_{0}+\beta \mu_{0}+\sum_{k=1}^{K}\left(\alpha_{k}+\beta \mu_{k}\right) x_{k, \tau}\right] d \tau\right.\right. \\
& \left.\left.+\beta\left(\gamma_{0}+\sum_{k=1}^{K} \gamma_{k} x_{k, \tau}\right)^{1 / 2} d w_{\tau}+u\left[\left(\mu_{0}+\sum_{k=1}^{K} \mu_{k} x_{k, \tau}\right) d \tau+\left(\gamma_{0}+\sum_{k=1}^{K} \gamma_{k} x_{k, \tau}\right)^{1 / 2} d w_{\tau}\right]\right)\right\} \\
& =\exp \left(u \log I_{t}\right) E_{t}\left\{\operatorname { e x p } \int _ { t } ^ { t + h } \left(\left[\alpha_{0}+(\beta+u) \mu_{0}+\sum_{k=1}^{K}\left(\alpha_{k}+(\beta+u) \mu_{k}\right) x_{k, \tau}\right] d \tau\right.\right. \\
& \left.\left.+(\beta+u)\left(\gamma_{0}+\sum_{k=1}^{K} \gamma_{k} x_{k, \tau}\right)^{1 / 2} d w_{\tau}\right)\right\} \\
& =\exp \left(u \log I_{t}\right) E_{t}\left\{\exp \int_{t}^{t+h}\left[\alpha_{0}+(\beta+u) \mu_{0}+\sum_{k=1}^{K}\left(\alpha_{k}+(\beta+u) \mu_{k}\right) x_{k, \tau}\right] d \tau\right. \\
& \left.\times E_{t}\left(\exp \left[(\beta+u) \int_{t}^{t+h}\left(\gamma_{0}+\sum_{k=1}^{K} \gamma_{k} x_{k, \tau}\right)^{1 / 2} d w_{\tau}\right] \mid \mathbb{X}_{k, \tau}\right)\right\}
\end{aligned}
$$

where $\mathbb{X}_{k, \tau}$ denotes the set $\left\{x_{k, \tau}\right\}_{\tau=t \cdots t+h}^{k=1 \cdots K}$.

Since $\exp \left[(\beta+u) \int_{t}^{t+h}\left(\gamma_{0}+\sum_{k=1}^{K} \gamma_{k} x_{k, \tau}\right)^{1 / 2} d w_{\tau}\right] \mid \mathbb{X}_{k, \tau}$

$$
\sim L N\left(0,(\beta+u)^{2} \int_{t}^{t+h}\left(\gamma_{0}+\sum_{k=1}^{K} \gamma_{k} x_{k, \tau}\right) d \tau\right)
$$

we deduce that: 


$$
\begin{aligned}
& C(t, t+h, u) \\
& =\exp \left(u \log I_{t}\right) E_{t}\left\{\exp \int_{t}^{t+h}\left[\alpha_{0}+(\beta+u) \mu_{0}+\sum_{k=1}^{K}\left(\alpha_{k}+(\beta+u) \mu_{k}\right) x_{k, \tau}\right] d \tau\right. \\
& \left.\times \exp \left[\frac{(\beta+u)^{2}}{2} \int_{t}^{t+h}\left(\gamma_{0}+\sum_{k=1}^{K} \gamma_{k} x_{k, \tau}\right) d \tau\right]\right\} \\
& =\exp \left(u \log I_{t}\right) E_{t}\left\{\operatorname { e x p } \int _ { t } ^ { t + h } \left(\left[\alpha_{0}+(\beta+u) \mu_{0}+\sum_{k=1}^{K}\left(\alpha_{k}+(\beta+u) \mu_{k}\right) x_{k, \tau}\right] d \tau\right.\right. \\
& \left.\left.+\frac{(\beta+u)^{2}}{2}\left(\gamma_{0}+\sum_{k=1}^{K} \gamma_{k} x_{k, \tau}\right) d \tau\right)\right\} \\
& =\exp \left(u \log I_{t}\right) \exp \int_{t}^{t+h}\left[\alpha_{0}+(\beta+u) \mu_{0}+\frac{(\beta+u)^{2}}{2} \gamma_{0}\right] d \tau \\
& \times E_{t}\left\{\exp \int_{t}^{t+h} \sum_{k=1}^{K}\left[\left(\alpha_{k}+(\beta+u) \mu_{k}+\frac{(\beta+u)^{2}}{2} \gamma_{k}\right) x_{k, \tau}\right] d \tau\right\} \\
& =\exp \left(u \log I_{t}\right) \exp \left\{h\left[\alpha_{0}+(\beta+u) \mu_{0}+\frac{(\beta+u)^{2}}{2} \gamma_{0}\right]\right\} \\
& \times E_{t}\left\{\exp \sum_{k=1}^{K} \int_{t}^{t+h}\left[\alpha_{k}+(\beta+u) \mu_{k}+\frac{(\beta+u)^{2}}{2} \gamma_{k}\right] x_{k, \tau} d \tau\right\} \\
& =\exp \left(u \log I_{t}\right) \exp \left\{h\left[\alpha_{0}+(\beta+u) \mu_{0}+\frac{(\beta+u)^{2}}{2} \gamma_{0}\right]\right\} \\
& \times \prod_{k=1}^{K} E_{t}\left\{\exp -\int_{t}^{t+h}-\left[\alpha_{k}+(\beta+u) \mu_{k}+\frac{(\beta+u)^{2}}{2} \gamma_{k}\right] x_{k, \tau} d \tau\right\}
\end{aligned}
$$

since factors $\left\{x_{k, t}\right\}, k=1, \cdots, K$ are independent,

$$
\begin{gathered}
=\exp \left(u \log I_{t}\right) \exp \left\{h\left[\alpha_{0}+(\beta+u) \mu_{0}+\frac{(\beta+u)^{2}}{2} \gamma_{0}\right]\right\} \\
\times \prod_{k=1}^{K} \exp \left[-H_{1}^{k}\left(h, z_{k}(u)\right) x_{k, t}-H_{2}^{k}\left(h, z_{k}(u)\right)\right]
\end{gathered}
$$

$$
\begin{array}{r}
=\exp \left(u \log I_{t}\right) \exp \left\{h\left[\alpha_{0}+(\beta+u) \mu_{0}+\frac{\gamma_{0}}{2}(\beta+u)^{2}\right]\right. \\
\left.-\sum_{k=1}^{K} H_{1}^{k}\left(h, z_{k}(u)\right) x_{k, t}-\sum_{k=1}^{K} H_{2}^{k}\left(h, z_{k}(u)\right)\right\},
\end{array}
$$

where

$$
z_{k}(u)=-\alpha_{k}-(\beta+u) \mu_{k}-\frac{\gamma_{k}}{2}(\beta+u)^{2},
$$


and $H_{1}^{k}(\cdot, \cdot)$ and $H_{2}^{k}(\cdot, \cdot)$ are given in equation(2.4)

\section{B Proof of Proposition 3}

The instantaneous interest rate is defined by:

$r(t)=\lim _{h \rightarrow 0}-\frac{1}{h} \log B(t, t+h)=\left.\frac{d[-\log B(t, t+h)]}{d h}\right|_{h=0}$.

We have:

$-\log B(t, t+h)=-h\left(\alpha_{0}+\beta \mu_{0}+\frac{\gamma_{0}}{2} \beta^{2}\right)+\sum_{k=1}^{K} H_{1}^{k}\left(h, z_{k}(0)\right) x_{k, t}+\sum_{k=1}^{K} H_{2}^{k}\left(h, z_{k}(0)\right)$.

We deduce that:

$\frac{d[-\log B(t, t+h)]}{d h}=-\alpha_{0}-\beta \mu_{0}-\frac{\gamma_{0}}{2} \beta^{2}+\sum_{k=1}^{K} \frac{d H_{1}^{k}\left(h, z_{k}(0)\right)}{d h} x_{k, t}+\sum_{k=1}^{K} \frac{d H_{2}^{k}\left(h, z_{k}(0)\right)}{d h}$,

where

$$
\begin{aligned}
H_{1}^{k}\left(h, z_{k}(0)\right)= & \frac{2 z_{k}(0)\left(\exp \left[\varepsilon_{k}\left(z_{k}(0)\right) h\right]-1\right)}{\left(\varepsilon_{k}\left(z_{k}(0)\right)+\xi_{k}\right)\left(\exp \left[\varepsilon_{k}\left(z_{k}(0)\right) h\right]-1\right)+2 \varepsilon_{k}\left(z_{k}(0)\right)}, \\
H_{2}^{k}\left(h, z_{k}(0)\right)= & \frac{-2 \xi_{k} \zeta_{k}}{\nu_{k}^{2}}\left\{\log \left[2 \varepsilon_{k}\left(z_{k}(0)\right)\right]+\frac{h}{2}\left[\varepsilon_{k}\left(z_{k}(0)\right)+\xi_{k}\right]\right. \\
& \left.\quad-\log \left[\left(\varepsilon_{k}\left(z_{k}(0)\right)+\xi_{k}\right)\left(\exp \left[\varepsilon_{k}\left(z_{k}(0)\right) h\right]-1\right)+2 \varepsilon_{k}\left(z_{k}(0)\right)\right]\right\} .
\end{aligned}
$$

Let us denote $\left(\varepsilon_{k}\left(z_{k}(0)\right)+\xi_{k}\right)\left(\exp \left[\varepsilon_{k}\left(z_{k}(0)\right) h\right]-1\right)+2 \varepsilon_{k}\left(z_{k}(0)\right) \equiv A$. We get:

$$
\begin{aligned}
& \left.\frac{d H_{1}^{k}\left(h, z_{k}(0)\right)}{d h}\right|_{h=0} \\
= & \frac{2 z_{k}(0) \exp \left[\varepsilon_{k}\left(z_{k}(0)\right) h\right] \varepsilon_{k}\left(z_{k}(0)\right) A}{A^{2}} \\
& \quad-\left.\frac{2 z_{k}(0)\left(\exp \left[\varepsilon_{k}\left(z_{k}(0)\right) h\right]-1\right)\left(\varepsilon_{k}\left(z_{k}(0)\right)+\xi_{k}\right) \exp \left[\varepsilon_{k}\left(z_{k}(0)\right) h\right] \varepsilon_{k}\left(z_{k}(0)\right)}{A^{2}}\right|_{h=0} \\
= & \frac{2 z_{k}(0) \varepsilon_{k}\left(z_{k}(0)\right) 2 \varepsilon_{k}\left(z_{k}(0)\right)-0}{\left[2 \varepsilon_{k}\left(z_{k}(0)\right)\right]^{2}}=z_{k}(0),
\end{aligned}
$$

and

$$
\begin{aligned}
& \left.\frac{d H_{2}^{k}\left(h, z_{k}(0)\right)}{d h}\right|_{h=0} \\
= & \left.\frac{-2 \xi_{k} \zeta_{k}}{\nu_{k}^{2}}\left\{\frac{1}{2}\left[\varepsilon_{k}\left(z_{k}(0)\right)+\xi_{k}\right]-\frac{1}{A}\left(\varepsilon_{k}\left(z_{k}(0)\right)+\xi_{k}\right) \exp \left[\varepsilon_{k}\left(z_{k}(0)\right) h\right] \varepsilon_{k}\left(z_{k}(0)\right)\right\}\right|_{h=0}
\end{aligned}
$$




$$
=\frac{-2 \xi_{k} \zeta_{k}}{\nu_{k}^{2}}\left\{\frac{1}{2}\left[\varepsilon_{k}\left(z_{k}(0)\right)+\xi_{k}\right]-\frac{\left(\varepsilon_{k}\left(z_{k}(0)\right)+\xi_{k}\right) \varepsilon_{k}\left(z_{k}(0)\right)}{2 \varepsilon_{k}\left(z_{k}(0)\right)}\right\}=0 .
$$

We deduce:

$$
r(t)=-\alpha_{0}-\beta \mu_{0}-\frac{\gamma_{0}}{2} \beta^{2}+\sum_{k=1}^{K} z_{k}(0) x_{k, t} .
$$

\section{Proof of Proposition 4}

Since

$$
E\left[\exp \left(\int_{t}^{t+h} d m_{\tau}\right)\left(f(t, t+h)-I_{t+h}\right)\right]=0
$$

we get:

$$
B(t, t+h) f(t, t+h)=E\left[\exp \left(\int_{t}^{t+h} d m_{\tau}\right) I_{t+h}\right]
$$

and

$$
f(t, t+h)=\frac{C(t, t+h, 1)}{C(t, t+h, 0)} .
$$

\section{Proof of Proposition 5}

$$
\begin{aligned}
& \text { Since } E_{t}\left[\int_{t}^{t+h}\left(\exp \int_{t}^{t+\tau} d m_{s}\right) d F_{\tau}\right]=0 \text {, we get: } \\
& F_{t, t+h} \\
= & E_{t}\left[\exp \left(\int_{t}^{t+h} d m_{\tau}\right) \exp \left(\int_{t}^{t+h} r_{\tau} d \tau\right) I_{t+h}\right] \\
= & E_{t}\left\{\exp \left[\int_{t}^{t+h}\left(d m_{\tau}+r_{\tau} d \tau\right)+\log I_{t}+\int_{t}^{t+h} d \log I_{\tau}\right]\right\} \\
= & I_{t} E_{t}\left[\exp \left(\int_{t}^{t+h}\left(d m_{\tau}+r_{\tau} d \tau+d \log I_{\tau}\right)\right]\right. \\
= & I_{t} E_{t}\left\{\operatorname { e x p } \int _ { t } ^ { t + h } \left[\left(\alpha_{0}+\beta \mu_{0}+\sum_{k=1}^{K}\left(\alpha_{k}+\beta \mu_{k}\right) x_{k, \tau}\right) d \tau\right.\right. \\
& \quad \beta\left(\gamma_{0}+\sum_{k=1}^{K} \gamma_{k} x_{k, \tau}\right)^{1 / 2} d w_{\tau}+\left(\mu_{0}+\sum_{k=1}^{K} \mu_{k} x_{k, \tau}\right) d \tau
\end{aligned}
$$




$$
\begin{aligned}
& \left.\left.\quad+\left(\gamma_{0}+\sum_{k=1}^{K} \gamma_{k} x_{k, \tau}\right)^{1 / 2} d w_{\tau}+\left(-\alpha_{0}-\beta \mu_{0}-\frac{\gamma_{0}}{2} \beta^{2}+\sum_{k=1}^{K} z_{k}(0) x_{k, \tau}\right) d \tau\right]\right\} \\
& =I_{t} E_{t}\left\{\operatorname { e x p } \int _ { t } ^ { t + h } \left[\left(\alpha_{0}+(\beta+1) \mu_{0}-\alpha_{0}-\beta \mu_{0}-\frac{\gamma_{0}}{2} \beta^{2}\right) d \tau\right.\right. \\
& \left.\left.+\sum_{k=1}^{K}\left(\alpha_{k}+\beta \mu_{k}+\mu_{k}+z_{k}(0)\right) x_{k, \tau} d \tau+(\beta+1)\left(\gamma_{0}+\sum_{k=1}^{K} \gamma_{k} x_{k, \tau}\right)^{1 / 2} d w_{\tau}\right]\right\} \\
& =I_{t} \exp \left[h\left(\mu_{0}-\frac{\gamma_{0}}{2} \beta^{2}\right)\right] E_{t}\left\{\exp \int_{t}^{t+h} \sum_{k=1}^{K}\left(\alpha_{k}+(\beta+1) \mu_{k}+z_{k}(0)\right) x_{k, \tau} d \tau\right. \\
& \left.\quad \times E_{t}\left[\exp \int_{t}^{t+h}(\beta+1)\left(\gamma_{0}+\sum_{k=1}^{K} \gamma_{k} x_{k, \tau}\right)^{1 / 2} d w_{\tau} \mid \mathbb{X}_{k, \tau}\right]\right\} .
\end{aligned}
$$

Since $\exp \int_{t}^{t+h}(\beta+1)\left(\gamma_{0}+\sum_{k=1}^{K} \gamma_{k} x_{k, \tau}\right)^{1 / 2} d w_{\tau} \mid \mathbb{X}_{k, \tau}$

$$
\sim \operatorname{LN}\left(0,(\beta+1)^{2} \int_{t}^{t+h}\left(\gamma_{0}+\sum_{k=1}^{K} \gamma_{k} x_{k, \tau}\right) d \tau\right)
$$

we get:

$$
\begin{aligned}
& F_{t, t+h} \\
& =I_{t} \exp \left[h\left(\mu_{0}-\frac{\gamma_{0}}{2} \beta^{2}\right)\right] \\
& \quad \times E_{t}\left\{\exp \int_{t}^{t+h}\left[\sum_{k=1}^{K}\left(\alpha_{k}+(\beta+1) \mu_{k}+z_{k}(0)\right) x_{k, \tau} d \tau+\frac{(\beta+1)^{2}}{2}\left(\gamma_{0}+\sum_{k=1}^{K} \gamma_{k} x_{k, \tau}\right) d \tau\right]\right\} \\
& =I_{t} \exp \left[h\left(\mu_{0}-\frac{\gamma_{0}}{2} \beta^{2}+\frac{(\beta+1)^{2}}{2} \gamma_{0}\right)\right] \\
& \quad \times E_{t}\left\{\exp \int_{t}^{t+h}\left[\sum_{k=1}^{K}\left(\alpha_{k}+(\beta+1) \mu_{k}+z_{k}(0)+\frac{(\beta+1)^{2}}{2} \gamma_{k}\right) x_{k, \tau}\right] d \tau\right\} \\
& =I_{t} \exp \left[h\left(\mu_{0}-\frac{\gamma_{0}}{2} \beta^{2}+\frac{(\beta+1)^{2}}{2} \gamma_{0}\right)\right] \\
& \quad \times \prod_{k=1}^{K} E_{t}\left\{\exp -\int_{t}^{t+h}-\left[\alpha_{k}+(\beta+1) \mu_{k}+z_{k}(0)+\frac{(\beta+1)^{2}}{2} \gamma_{k}\right] x_{k, \tau} d \tau\right\} \\
& =I_{t} \exp \left[h\left(\mu_{0}-\frac{\gamma_{0}}{2} \beta^{2}+\frac{(\beta+1)^{2}}{2} \gamma_{0}\right)\right] \prod_{k=1}^{K} \exp \left[-H_{1}^{k}\left(h, l_{k}\right) x_{k, t}-H_{2}^{k}\left(h, l_{k}\right)\right] \\
& =I_{t} \exp \left[h\left(\mu_{0}+\frac{1+2 \beta}{2} \gamma_{0}\right)-\sum_{k=1}^{K} H_{1}^{k}\left(h, l_{k}\right) x_{k, t}-\sum_{k=1}^{K} H_{2}^{k}\left(h, l_{k}\right)\right],
\end{aligned}
$$


where

$$
\begin{aligned}
l_{k} & =-\alpha_{k}-(\beta+1) \mu_{k}-z_{k}(0)-\frac{\gamma_{k}}{2}(\beta+1)^{2} \\
& =-\mu_{k}-\frac{1+2 \beta}{2} \gamma_{k} .
\end{aligned}
$$

\section{E Proof of Proposition 6}

Let us first consider the call option with price $G(t, t+h, X)$. Its price is given by:

$$
\begin{aligned}
& G(t, t+h, X) \\
&= E_{t}\left\{\exp \left(\int_{t}^{t+h} d m_{\tau}\right)\left[\exp \left(\log I_{t+h}\right)-X\right]^{+}\right\} \\
&= E_{t}\left\{\exp \left(\int_{t}^{t+h} d m_{\tau}\right)\left[\exp \left(\log I_{t+h}\right)-X\right] \mathbf{1}_{\left.-\log I_{t+h} \leq-\log X\right\}}\right. \\
&= A_{1,-1}\left(-\log X ; x_{1, t}, \cdots, x_{K, t}, \log I_{t}, h\right)-X A_{0,-1}\left(-\log X ; x_{1, t}, \cdots, x_{K, t}, \log I_{t}, h\right), \\
& \text { where } A_{a, b}\left(y ; x_{1, t}, \cdots, x_{K, t}, \log I_{t}, h\right)=E_{t}\left[\exp \left(\int_{t}^{t+h} d m_{\tau}\right) \exp \left(a \log I_{t+h}\right) 1_{b \log I_{t+h} \leq y}\right] .
\end{aligned}
$$

The Fourier-Stieltjes transform of $A_{a, b}\left(y ; x_{1, t}, \cdots, x_{K, t}, \log I_{t}, h\right)$ is

$$
\begin{aligned}
& \int_{\Re} \exp (i v y) d A_{a, b}\left(y ; x_{1, t}, \cdots, x_{K, t}, \log I_{t}, h\right) \\
= & E_{t}\left\{\exp \left(\int_{t}^{t+h} d m_{\tau}\right) \exp \left[(a+i v b) \log I_{t+h}\right]\right\}=C(t, t+h, a+i v b) .
\end{aligned}
$$

We deduce that:

$$
\begin{aligned}
& A_{a, b}\left(y ; x_{1, t}, \cdots, x_{K, t}, \log I_{t}, h\right) \\
= & \frac{C(t, t+h, a)}{2}-\frac{1}{\pi} \int_{0}^{\infty} \frac{\operatorname{Im}[C(t, t+h, a+i v b) \exp (-i v y)]}{v} d v
\end{aligned}
$$

[see Duffie, Pan and Singleton (2000), p1352].

By substitution we get the call price

$$
\begin{aligned}
G(t, t+h, X)= & \frac{C(t, t+h, 1)}{2}-\frac{1}{\pi} \int_{0}^{\infty} \frac{\operatorname{Im}[C(t, t+h, 1-i v) \exp (i v \log X)]}{v} d v \\
& -X\left\{\frac{C(t, t+h, 0)}{2}-\frac{1}{\pi} \int_{0}^{\infty} \frac{\operatorname{Im}[C(t, t+h,-i v) \exp (i v \log X)]}{v} d v\right\} .
\end{aligned}
$$


Similarly, for the put option with price $H(t, t+h, X)$, we have

$$
\begin{aligned}
& H(t, t+h, X) \\
= & E_{t}\left\{\exp \left(\int_{t}^{t+h} d m_{\tau}\right)\left[X-\exp \left(\log I_{t+h}\right)\right]^{+}\right\} \\
= & E_{t}\left\{\exp \left(\int_{t}^{t+h} d m_{\tau}\right)\left[X-\exp \left(\log I_{t+h}\right)\right] \mathbf{1}_{\log I_{t+h} \leq \log X}\right\} \\
=- & A_{1,1}\left(\log X ; x_{1, t}, \cdots, x_{K, t}, \log I_{t}, h\right)+X A_{0,1}\left(\log X ; x_{1, t}, \cdots, x_{K, t}, \log I_{t}, h\right) \\
=- & \frac{C(t, t+h, 1)}{2}+\frac{1}{\pi} \int_{0}^{\infty} \frac{\operatorname{Im}[C(t, t+h, 1+i v) \exp (-i v \log X)]}{v} d v \\
& \quad+X\left\{\frac{C(t, t+h, 0)}{2}-\frac{1}{\pi} \int_{0}^{\infty} \frac{\operatorname{Im}[C(t, t+h, i v) \exp (-i v \log X)]}{v} d v\right\} .
\end{aligned}
$$

\section{F $\quad$ Proof of Proposition 7}

We have:

$$
\begin{aligned}
& F_{t+h, t+h+m}=I_{t+h} \exp \left[m\left(\mu_{0}-\frac{1+2 \beta}{2} \gamma_{0}\right)-\sum_{k=1}^{K} H_{1}^{k}\left(m, l_{k}\right) x_{k, t+h}-\sum_{k=1}^{K} H_{2}^{k}\left(m, l_{k}\right)\right], \\
& \log F_{t+h, t+h+m}=\log I_{t+h}+m\left(\mu_{0}-\frac{1+2 \beta}{2} \gamma_{0}\right)-\sum_{k=1}^{K} H_{1}^{k}\left(m, l_{k}\right) x_{k, t+h}-\sum_{k=1}^{K} H_{2}^{k}\left(m, l_{k}\right) .
\end{aligned}
$$

Therefore,

$$
\begin{aligned}
& C^{F}(t, t+h, t+h+m, u) \\
= & E_{t}\left(\exp \int_{t}^{t+h} d m_{\tau}\left(F_{t+h, t+h+m}\right)^{u}\right) \\
= & E_{t}\left[\exp \int_{t}^{t+h} d m_{\tau} \exp \left(u \log F_{t+h, t+h+m}\right)\right] \\
= & E_{t}\left[\exp \left(\int_{t}^{t+h} d m_{\tau}+u \log F_{t+h, t+h+m}\right)\right] \\
= & E_{t}\left\{\operatorname { e x p } \left(\int_{t}^{t+h}\left[\left(\alpha_{0}+\beta \mu_{0}+\sum_{k=1}^{K}\left(\alpha_{k}+\beta \mu_{k}\right) x_{k, \tau}\right) d \tau+\beta\left(\gamma_{0}+\sum_{k=1}^{K} \gamma_{k} x_{k, \tau}\right)^{1 / 2} d w_{\tau}\right]\right.\right.
\end{aligned}
$$




$$
\begin{aligned}
& +u\left[\log I_{t}+\int_{t}^{t+h} d \log I_{t}+m\left(\mu_{0}-\frac{1+2 \beta}{2} \gamma_{0}\right)\right. \\
& \left.\left.\left.-\sum_{k=1}^{K} H_{1}^{k}\left(m, l_{k}\right)\left(x_{k, t}+\int_{t}^{t+h} d x_{k, \tau}\right)-\sum_{k=1}^{K} H_{2}^{k}\left(m, l_{k}\right)\right]\right)\right\} \\
& =\exp \left[u \log I_{t}+u m\left(\mu_{0}-\frac{1+2 \beta}{2} \gamma_{0}\right)-u \sum_{k=1}^{K} H_{1}^{k}\left(m, l_{k}\right) x_{k, t}-u \sum_{k=1}^{K} H_{2}^{k}\left(m, l_{k}\right)\right] \\
& \times E_{t}\left\{\operatorname { e x p } \int _ { t } ^ { t + h } \left[\left(\alpha_{0}+\beta \mu_{0}+\sum_{k=1}^{K}\left(\alpha_{k}+\beta \mu_{k}\right) x_{k, \tau}\right) d \tau+\beta\left(\gamma_{0}+\sum_{k=1}^{K} \gamma_{k} x_{k, \tau}\right)^{1 / 2} d w_{\tau}\right.\right. \\
& +u\left(\mu_{0}+\sum_{k=1}^{K} \mu_{k} x_{k, \tau}\right) d \tau+u\left(\gamma_{0}+\sum_{k=1}^{K} \gamma_{k} x_{k, \tau}\right)^{1 / 2} d w_{\tau} \\
& \left.\left.-u \sum_{k=1}^{K} H_{1}^{k}\left(m, l_{k}\right)\left(\xi_{k}\left(\zeta_{k}-x_{k, \tau}\right) d \tau+\nu_{k} \sqrt{x_{k, \tau}} d w_{k, \tau}\right)\right]\right\} \\
& =\exp \left[u \log I_{t}+u m\left(\mu_{0}-\frac{1+2 \beta}{2} \gamma_{0}\right)-u \sum_{k=1}^{K} H_{1}^{k}\left(m, l_{k}\right) x_{k, t}-u \sum_{k=1}^{K} H_{2}^{k}\left(m, l_{k}\right)\right] \\
& \times E_{t}\left\{\operatorname { e x p } \int _ { t } ^ { t + h } \left[\left(\alpha_{0}+\beta \mu_{0}+u \mu_{0}-u \sum_{k=1}^{K} H_{1}^{k}\left(m, l_{k}\right) \xi_{k} \zeta_{k}\right) d \tau\right.\right. \\
& \left.+\sum_{k=1}^{K}\left(\alpha_{k}+\beta \mu_{k}+u \mu_{k}+u H_{1}^{k}\left(m, l_{k}\right) \xi_{k}\right) x_{k, \tau}\right) d \tau \\
& \left.\left.+(\beta+u)\left(\gamma_{0}+\sum_{k=1}^{K} \gamma_{k} x_{k, \tau}\right)^{1 / 2} d w_{\tau}-u \sum_{k=1}^{K} H_{1}^{k}\left(m, l_{k}\right) \nu_{k} \sqrt{x_{k, \tau}} d w_{k, \tau}\right]\right\} \\
& =\exp \left\{u\left[\log I_{t}+m\left(\mu_{0}-\frac{1+2 \beta}{2} \gamma_{0}\right)-\sum_{k=1}^{K} H_{1}^{k}\left(m, l_{k}\right) x_{k, t}-\sum_{k=1}^{K} H_{2}^{k}\left(m, l_{k}\right)\right]\right. \\
& \left.+h\left[\alpha_{0}+(\beta+u) \mu_{0}-u \sum_{k=1}^{K} H_{1}^{k}\left(m, l_{k}\right) \xi_{k} \zeta_{k}\right]\right\} \\
& \times E_{t}\left\{\exp \left(\int_{t}^{t+h} \sum_{k=1}^{K}\left[\alpha_{k}+(\beta+u) \mu_{k}+u H_{1}^{k}\left(m, l_{k}\right) \xi_{k}\right] x_{k, \tau} d \tau\right)\right. \\
& \left.\times E_{t}\left(\exp \int_{t}^{t+h}\left[(\beta+u)\left(\gamma_{0}+\sum_{k=1}^{K} \gamma_{k} x_{k, \tau}\right)^{1 / 2} d w_{\tau}-u \sum_{k=1}^{K} H_{1}^{k}\left(m, l_{k}\right) \nu_{k} \sqrt{x_{k, \tau}} d w_{k, \tau}\right] \mid \mathbb{X}_{k, \tau}\right)\right\} \\
& =\exp \left\{u \log I_{t}+(u m+h \beta+h u) \mu_{0}-\frac{1+2 \beta}{2} u m \gamma_{0}+h \alpha_{0}\right.
\end{aligned}
$$




$$
\begin{aligned}
& \left.-\sum_{k=1}^{K} u H_{1}^{k}\left(m, l_{k}\right)\left(x_{k, t}+h \xi_{k} \zeta_{k}\right)-\sum_{k=1}^{K} u H_{2}^{k}\left(m, l_{k}\right)\right\} \\
& \times E_{t}\left\{\exp \left(\int_{t}^{t+h} \sum_{k=1}^{K}\left[\alpha_{k}+(\beta+u) \mu_{k}+u H_{1}^{k}\left(m, l_{k}\right) \xi_{k}\right] x_{k, \tau} d \tau\right)\right. \\
& \left.\times\left(\exp \int_{t}^{t+h}\left(\frac{(\beta+u)^{2}}{2}\left(\gamma_{0}+\sum_{k=1}^{K} \gamma_{k} x_{k, \tau}\right)+\sum_{k=1}^{K} \frac{u^{2}}{2}\left[H_{1}^{k}\left(m, l_{k}\right)\right]^{2} \nu_{k}^{2} x_{k, \tau}\right) d \tau\right)\right\} \\
& =\exp \left\{u \log I_{t}+(u m+h \beta+h u) \mu_{0}-\frac{1+2 \beta}{2} u m \gamma_{0}+h \alpha_{0}\right. \\
& \left.-\sum_{k=1}^{K} u H_{1}^{k}\left(m, l_{k}\right)\left(x_{k, t}+h \xi_{k} \zeta_{k}\right)-\sum_{k=1}^{K} u H_{2}^{k}\left(m, l_{k}\right)\right\} \\
& \times E_{t}\left\{\operatorname { e x p } \int _ { t } ^ { t + h } \left(\frac{(\beta+u)^{2}}{2} \gamma_{0}\right.\right. \\
& \left.\left.+\sum_{k=1}^{K}\left[\alpha_{k}+(\beta+u) \mu_{k}+u H_{1}^{k}\left(m, l_{k}\right) \xi_{k}+\frac{(\beta+u)^{2}}{2} \gamma_{k}+\frac{u^{2}}{2}\left[H_{1}^{k}\left(m, l_{k}\right)\right]^{2} \nu_{k}^{2}\right] x_{k, \tau}\right) d \tau\right\} \\
& =\exp \left\{u \log I_{t}+(u m+h \beta+h u) \mu_{0}+\frac{(\beta+u)^{2} h-(1+2 \beta) u m}{2} \gamma_{0}+h \alpha_{0}\right. \\
& \left.-\sum_{k=1}^{K} u H_{1}^{k}\left(m, l_{k}\right)\left(x_{k, t}+h \xi_{k} \zeta_{k}\right)-\sum_{k=1}^{K} u H_{2}^{k}\left(m, l_{k}\right)\right\} \\
& \times \prod_{k=1}^{K} E_{t}\left[\exp -\int_{t}^{t+h}-\left(\alpha_{k}+(\beta+u) \mu_{k}+u H_{1}^{k}\left(m, l_{k}\right) \xi_{k}\right.\right. \\
& \left.\left.+\frac{(\beta+u)^{2}}{2} \gamma_{k}+\frac{u^{2}}{2}\left[H_{1}^{k}\left(m, l_{k}\right)\right]^{2} \nu_{k}^{2}\right) x_{k, \tau} d \tau\right] \\
& =\exp \left\{u \log I_{t}+(u m+h \beta+h u) \mu_{0}+\frac{(\beta+u)^{2} h-(1+2 \beta) u m}{2} \gamma_{0}+h \alpha_{0}\right. \\
& \left.-\sum_{k=1}^{K} u H_{1}^{k}\left(m, l_{k}\right)\left(x_{k, t}+h \xi_{k} \zeta_{k}\right)-\sum_{k=1}^{K} u H_{2}^{k}\left(m, l_{k}\right)\right\} \\
& \times \prod_{k=1}^{K} \exp \left[-H_{1}^{k}\left(h, p_{k}(m, u)\right) x_{k, t}-H_{2}^{k}\left(h, p_{k}(m, u)\right)\right] \\
& =\exp \left\{u \log I_{t}+(u m+h \beta+h u) \mu_{0}+\frac{(\beta+u)^{2} h-(1+2 \beta) u m}{2} \gamma_{0}+h \alpha_{0}\right. \\
& -\sum_{k=1}^{K} u H_{1}^{k}\left(m, l_{k}\right) h \xi_{k} \zeta_{k}-\sum_{k=1}^{K} u H_{2}^{k}\left(m, l_{k}\right)-\sum_{k=1}^{K} H_{2}^{k}\left(h, p_{k}(m, u)\right) \\
& \left.-\sum_{k=1}^{K}\left[u H_{1}^{k}\left(m, l_{k}\right)+H_{1}^{k}\left(h, p_{k}(m, u)\right)\right] x_{k, t}\right\}
\end{aligned}
$$




$$
\begin{aligned}
= & \exp \left(u \log I_{t}\right) \exp \left\{m\left(u \mu_{0}-\frac{1+2 \beta}{2} u \gamma_{0}\right)+h\left[(\beta+u) \mu_{0}+\frac{(\beta+u)^{2}}{2} \gamma_{0}+\alpha_{0}\right]\right. \\
& -\sum_{k=1}^{K} u H_{1}^{k}\left(m, l_{k}\right) h \xi_{k} \zeta_{k}-\sum_{k=1}^{K} u H_{2}^{k}\left(m, l_{k}\right)-\sum_{k=1}^{K} H_{2}^{k}\left(h, p_{k}(m, u)\right) \\
& \left.-\sum_{k=1}^{K}\left[u H_{1}^{k}\left(m, l_{k}\right)+H_{1}^{k}\left(h, p_{k}(m, u)\right)\right] x_{k, t}\right\},
\end{aligned}
$$

where

$$
p_{k}(m, u)=-\alpha_{k}-(\beta+u) \mu_{k}-u H_{1}^{k}\left(m, l_{k}\right) \xi_{k}-\frac{\gamma_{k}}{2}(\beta+u)^{2}-\frac{u^{2}}{2}\left[H_{1}^{k}\left(m, l_{k}\right)\right]^{2} \nu_{k}^{2},
$$

$l_{k}$ is given in equation $(2.10)$, and $H_{1}^{k}(\cdot, \cdot)$ and $H_{2}^{k}(\cdot, \cdot)$ are given in equation(2.4).

\section{G Proof of Proposition 8}

The price of the call optioin written on the futures is:

$$
\begin{aligned}
& G^{F}(t, t+h, t+h+m, X) \\
= & E_{t}\left\{\exp \left(\int_{t}^{t+h} d m_{\tau}\right)\left[\exp \left(\log F_{t+h, t+h+m}\right)-X\right]^{+}\right\} \\
= & E_{t}\left\{\exp \left(\int_{t}^{t+h} d m_{\tau}\right)\left[\exp \left(\log F_{t+h, t+h+m}\right)-X\right] \mathbf{1}_{-\log F_{t+h, t+h+m} \leq-\log X}\right\} \\
= & A_{1,-1}\left(-\log X ; x_{1, t}, \cdots, x_{K, t}, \log F_{t, t+h+m}, h\right) \\
& \quad-X A_{0,-1}\left(-\log X ; x_{1, t}, \cdots, x_{K, t}, \log F_{t, t+h+m}, h\right),
\end{aligned}
$$

where

$$
\begin{aligned}
& A_{a, b}\left(y ; x_{1, t}, \cdots, x_{K, t}, \log F_{t, t+h+m}, h\right) \\
= & E_{t}\left[\exp \left(\int_{t}^{t+h} d m_{\tau}\right) \exp \left(a \log F_{t+h, t+h+m}\right) 1_{b \log F_{t+h, t+h+m} \leq y}\right] .
\end{aligned}
$$

The Fourier-Stieltjes transform of $A_{a, b}\left(y ; x_{1, t}, \cdots, x_{K, t}, \log F_{t, t+h+m}, h\right)$ is:

$$
\begin{aligned}
& \int_{\Re} \exp (i v y) d A_{a, b}\left(y ; x_{1, t}, \cdots, x_{K, t}, \log F_{t, t+h+m}, h\right) \\
= & E_{t}\left\{\exp \left(\int_{t}^{t+h} d m_{\tau}\right) \exp \left[(a+i v b) \log F_{t+h, t+h+m}\right]\right\}=C^{F}(t, t+h, t+h+m, a+i v b) .
\end{aligned}
$$


Therefore, we have:

$$
\begin{aligned}
& A_{a, b}\left(y ; x_{1, t}, \cdots, x_{K, t}, \log F_{t, t+h+m}, h\right) \\
= & \frac{C^{F}(t, t+h, t+h+m, a)}{2}-\frac{1}{\pi} \int_{0}^{\infty} \frac{\operatorname{Im}\left[C^{F}(t, t+h, t+h+m, a+i v b) \exp (-i v y)\right]}{v} d v,
\end{aligned}
$$

and

$$
\begin{aligned}
& G^{F}(t, t+h, t+h+m, X) \\
= & \frac{C^{F}(t, t+h, t+h+m, 1)}{2}-\frac{1}{\pi} \int_{0}^{\infty} \frac{\operatorname{Im}\left[C^{F}(t, t+h, t+h+m, 1-i v) \exp (i v \log X)\right]}{v} d v \\
& -X\left\{\frac{C^{F}(t, t+h, t+h+m, 0)}{2}\right. \\
& \left.-\frac{1}{\pi} \int_{0}^{\infty} \frac{\operatorname{Im}\left[C^{F}(t, t+h, t+h+m,-i v) \exp (i v \log X)\right]}{v} d v\right\} .
\end{aligned}
$$

\section{H Proof of Proposition 9}

The first restriction in Equation (3.2) holds, if and only, if

$$
z_{k}(1)=-\alpha_{k}-(\beta+1) \mu_{k}-\frac{\gamma_{k}}{2}(\beta+1)^{2}=0, \forall k=1, \cdots, K
$$

This implies $\varepsilon_{k}\left(z_{k}(1)\right)=\left|\xi_{k}\right|, \forall k=1, \cdots, K$, and

$$
\begin{aligned}
H_{2}^{k}\left(h, z_{k}(1)\right) & =\frac{-2 \xi_{k} \zeta_{k}}{\nu_{k}^{2}}\left\{\log \left|2 \xi_{k}\right|+\frac{h}{2}\left(\left|\xi_{k}\right|+\xi_{k}\right)-\log \left[\left(\left|\xi_{k}\right|+\xi_{k}\right)\left(\exp \left(\left|\xi_{k}\right| h\right)-1\right)+2\left|\xi_{k}\right|\right]\right\} \\
& =0, \text { no matter if } \xi_{k}>0 \text { or } \xi_{k}<0, \forall k=1, \cdots, K
\end{aligned}
$$

This, with the second restriction in Equation (3.2), implies that

$$
\alpha_{0}+(\beta+1) \mu_{0}+\frac{\gamma_{0}}{2}\left(\beta_{1}\right)^{2}=0
$$

Therefore, Equation (3.2) is equivalent to

$$
\alpha_{k}+(\beta+1) \mu_{k}+\frac{\gamma_{k}}{2}\left(\beta_{1}\right)^{2}=0, \quad \forall k=0, \cdots, K
$$




\section{Figures and Tables}


Figure 1: Plots of Observations

This figure plots the observations for estimation in this paper. The data ranges from Jan 3, 2001 to Dec 29, 2006 and there are 1,506 days in total. On each day, there are six observations including the daily log difference of S\&P 500 Index (dlogSPXspot), annualized log of futures spot ratio (ALFSR) for two S\&P 500 futures, and price-vega ratio for three S\&P 500 options.
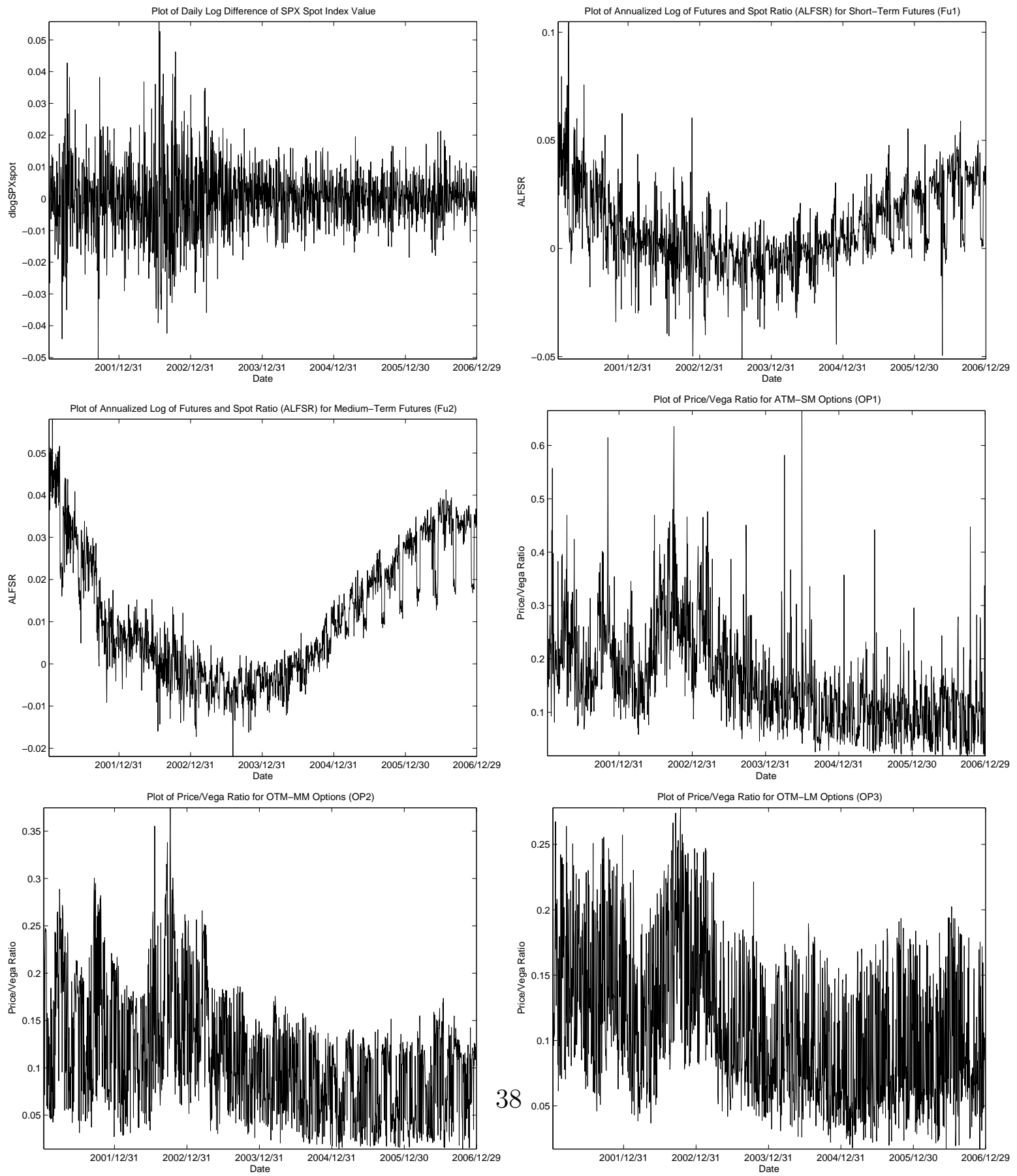
Figure 2: In-Sample Absolute Percentage Pricing (Forecasting) Errors from the OneFactor Model

This figure plots the in-sample pricing (forecasting) errors from the one-factor model. The pricing errors are measured as the absolute difference between the model implied price and the observed price as a percentage of the observed price. For the index, we report on the $\log$ index value, while for the futures and options, the actual prices are examined. There are 1,506 days in the dataset.
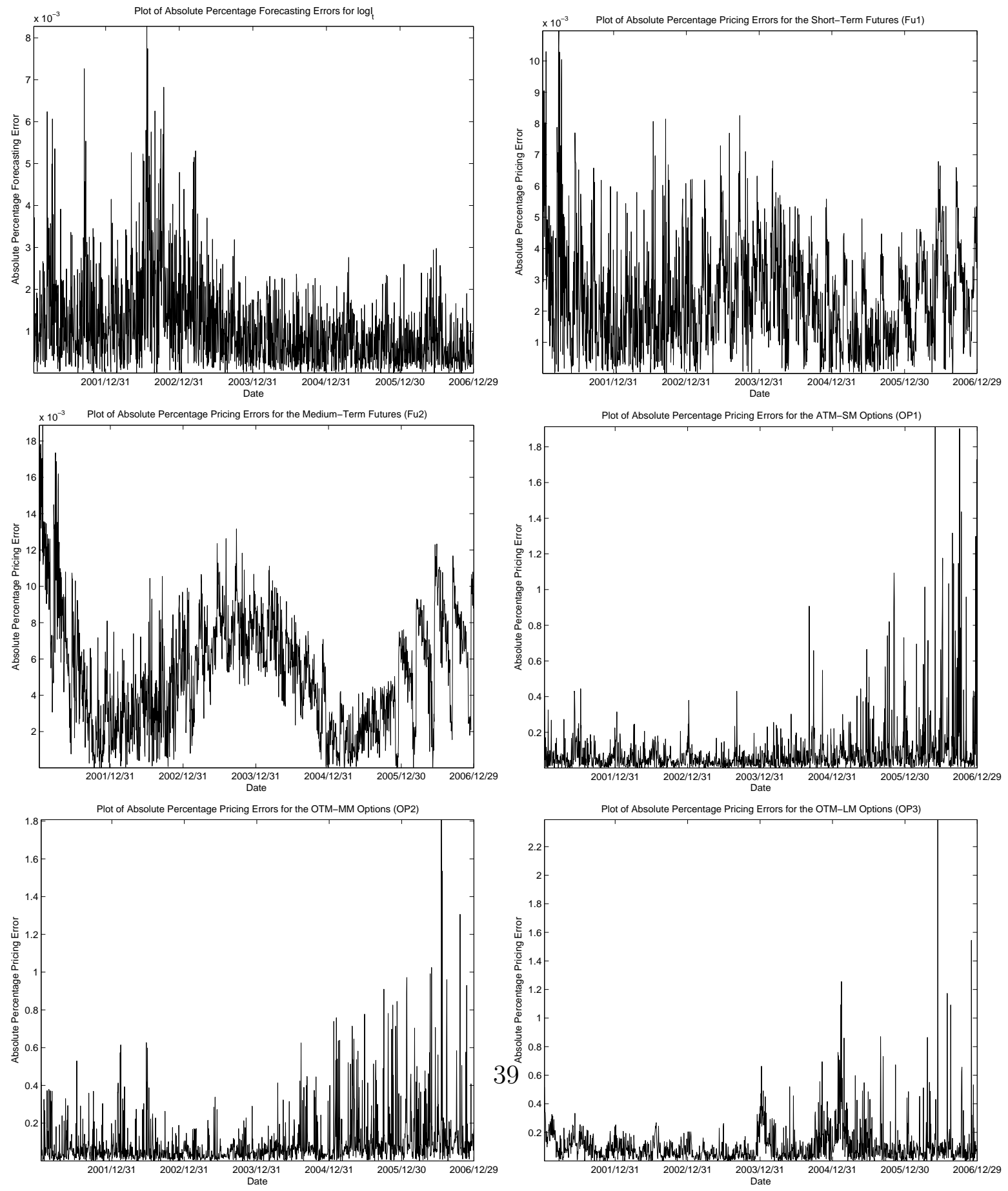
Figure 3: In-Sample Absolute Percentage Pricing (Forecasting) Errors from the TwoFactor Model

This figure plots the in-sample pricing (forecasting) errors from the two-factor model. The pricing errors are measured as the absolute difference between the model implied price and the observed price as a percentage of the observed price. For the index, we report on the $\log$ index value, while for the futures and options, the actual prices are examined. There are 1,506 days in the dataset.
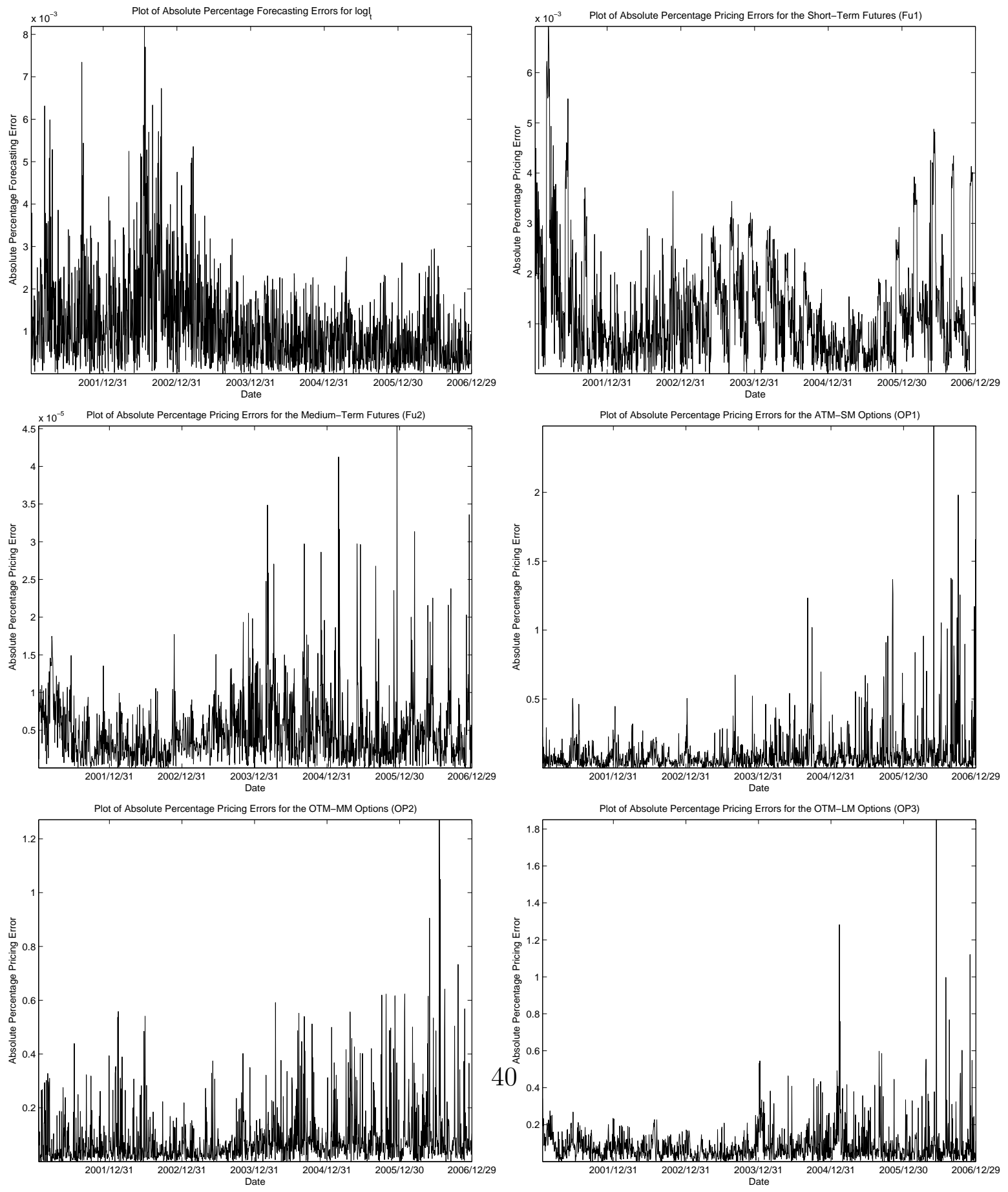
Figure 4: In-Sample Absolute Percentage Pricing (Forecasting) Errors from the ThreeFactor Model

This figure plots the in-sample pricing (forecasting) errors from the three-factor model. The pricing errors are measured as the absolute difference between the model implied price and the observed price as a percentage of the observed price. For the index, we report on the $\log$ index value, while for the futures and options, the actual prices are examined. There are 1,506 days in the dataset.
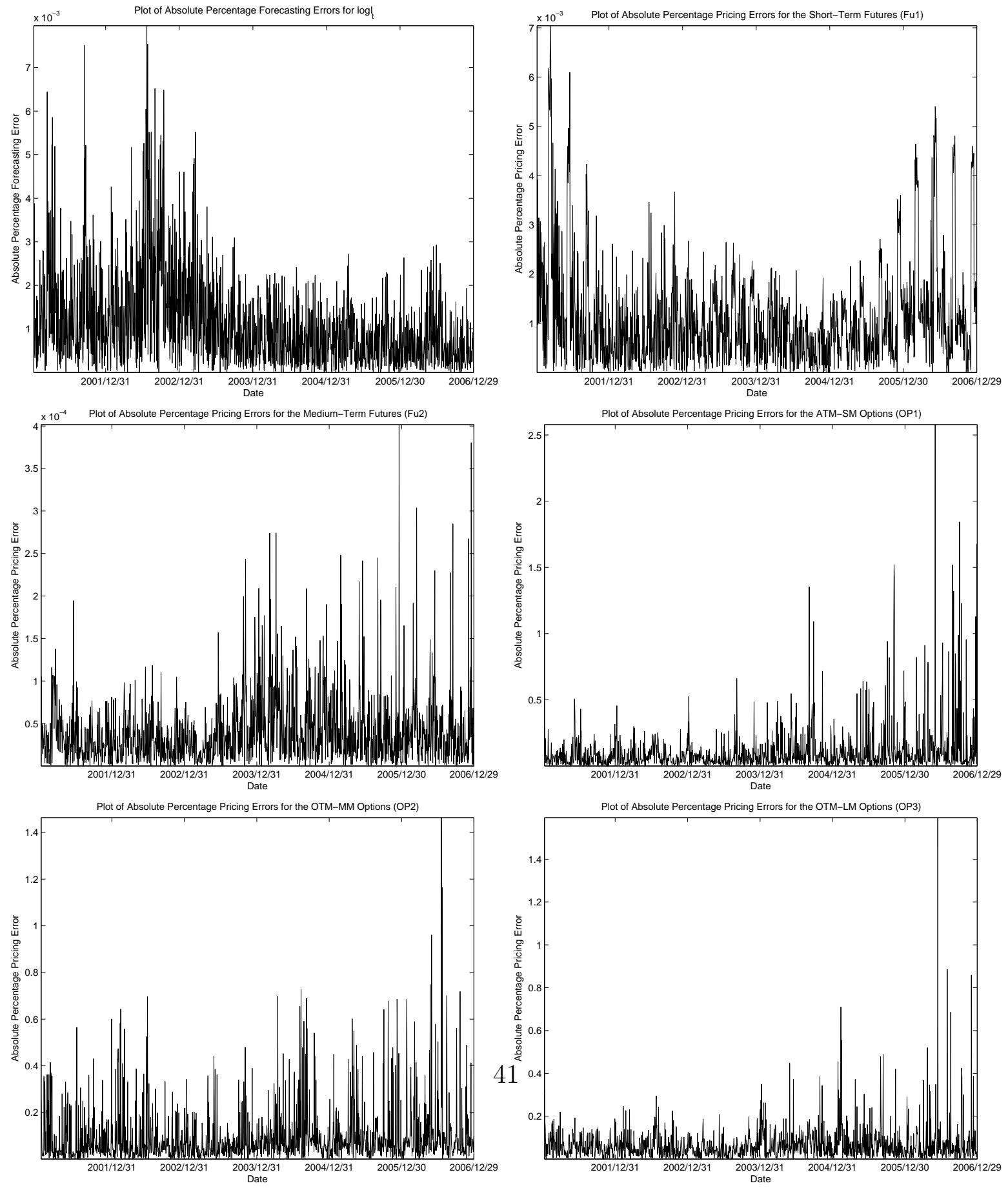
Figure 5: Filtered Factors from the Factor Models

This figure plots the filtered underly factors $\left\{\hat{x}_{k t}\right\}$ for each model, based on the parameter estimate in this paper.

Factor Implied from the One-Factor Model

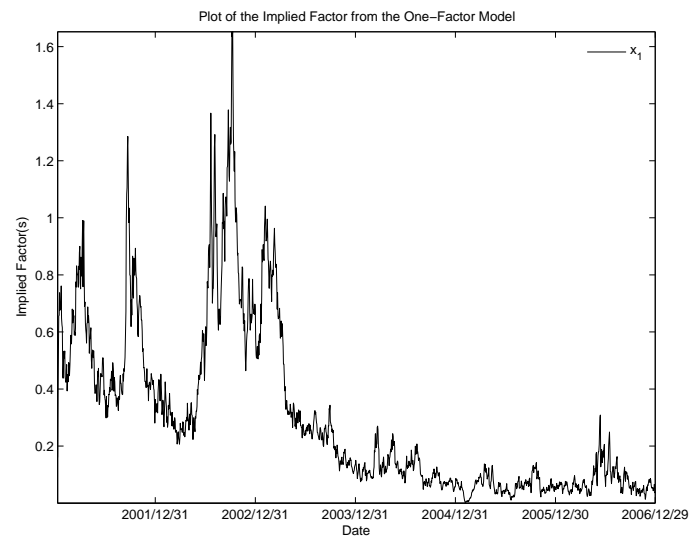

Factors Implied from the Two-Factor Model

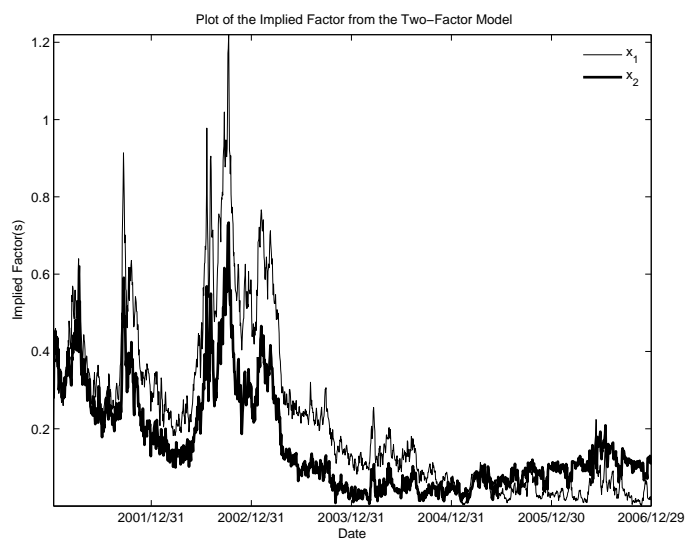

Factors Implied from the Three-Factor Model

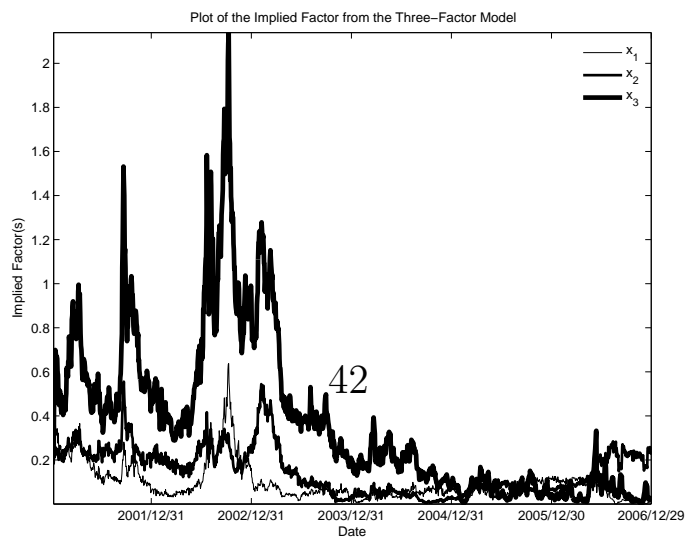


Table 1: Summary Statistics for the Data

This table summarizes statistics for the daily data ranging from January 3, 2001 to December 29, 2006. It covers the spot S\&P 500 Index (SPX), the two index futures (Fu1 and Fu2), and the three index options (Op1 to Op3) used for estimation, and the options used for out-of-the-sample test. The daily log difference of S\&P 500 Index is reported on the first row. For the futures, statistics for time-to-maturity (T2M), annualized log of futures spot ratio (ALFSR) and total number of outstanding futures contract (open interest) are presented. Fu1 refers to the short-term futures with the shortest maturity and Fu2 refers to the medium-term futures expiring a quarter later than Fu1. For the options, statistics for time-to-maturity (T2M), moneyness, price-vega ratio, trading volume and open interest are shown in the table. Op1 represents at-themoney options with short maturity (ATM-SM). Op2 stands for out-of-the-money options with medium maturity (OTM-MM), and Op3 are out-of-the-money options with long maturity (OTM-LM). The last panel summarizes the out-of-the sample options.

\begin{tabular}{lrrrrrrr}
\hline Variable & Mean & Std.Dev. & Skewness & Kurtosis & Min & Median & Max \\
\hline In Sample & & & & & & & \\
dlogSPX & 0.000 & 0.011 & 0.161 & 5.844 & -0.050 & 0.000 & 0.056 \\
\hline In Sample & & & & & & & \\
Fu1 T2M (years) & 0.172 & 0.0721 & 0.0142 & 1.817 & 0.041 & 0.173 & 0.307 \\
Fu1 ALFSR & 0.009 & 0.018 & 0.474 & 3.910 & -0.051 & 0.005 & 0.105 \\
Fu1 Open Int & 520,907 & 128,143 & -1.520 & 4.905 & 71,345 & 564,230 & 669,216 \\
\hline In Sample & & & & & & & \\
Fu2 T2M (years) & 0.423 & 0.072 & 0.017 & 1.820 & 0.288 & 0.425 & 0.559 \\
Fu2 ALFSR & 0.011 & 0.015 & 0.481 & 2.218 & -0.022 & 0.008 & 0.058 \\
Fu2 Open Int & 85,164 & 140,107 & 2.440 & 8.105 & 1,744 & 25,647 & 665,176 \\
\hline
\end{tabular}

(to be continued on next page...) 
Table 1: (continued)

\begin{tabular}{|c|c|c|c|c|c|c|c|}
\hline Variable & Mean & Std.Dev. & Skewness & Kurtosis & Min & Median & Max \\
\hline \multicolumn{8}{|l|}{ In Sample } \\
\hline Op1 T2M (years) & 0.081 & 0.034 & 0.398 & 2.476 & 0.027 & 0.082 & 0.162 \\
\hline Op1 Moneyness & 0.994 & 0.012 & 0.149 & 2.677 & 0.970 & 0.995 & 1.030 \\
\hline Op1 Price/Vega & 0.160 & 0.093 & 1.173 & 5.109 & 0.018 & 0.143 & 0.665 \\
\hline Op1 Trading vol & 7,467 & 6,353 & 2.840 & 15.921 & 239 & 5,853 & 59,702 \\
\hline Op1 Open int & 31,716 & 27,922 & 1.696 & 6.587 & 143 & 24,269 & 191,634 \\
\hline \multicolumn{8}{|l|}{ In Sample } \\
\hline Op2 T2M (years) & 0.266 & 0.076 & 0.675 & 2.468 & 0.164 & 0.244 & 0.490 \\
\hline Op2 Moneyness & 0.959 & 0.036 & -0.812 & 2.703 & 0.851 & 0.968 & 1.000 \\
\hline Op2 Price/Vega & 0.110 & 0.064 & 0.811 & 3.418 & 0.014 & 0.104 & 0.375 \\
\hline Op2 Trading vol & 3,963 & 3,921 & 3.130 & 25.210 & 6 & 2,700 & 54,275 \\
\hline Op2 Open int & 17,892 & 16,759 & 1.546 & 5.638 & 100 & 12,752 & 94,890 \\
\hline \multicolumn{8}{|l|}{ In Sample } \\
\hline Op3 T2M (years) & 0.810 & 0.235 & 0.875 & 3.056 & 0.501 & 0.753 & 1.479 \\
\hline Op3 Moneyness & 0.931 & 0.043 & -0.046 & 1.832 & 0.850 & 0.930 & 1.000 \\
\hline Op3 Price/Vega & 0.111 & 0.056 & 0.557 & 2.492 & 0.017 & 0.103 & 0.278 \\
\hline Op3 Trading vol & 1,627 & 1,820 & 3.556 & 25.661 & 1 & 1,050 & 21,000 \\
\hline Op3 Open int & 11,766 & $10,091.15$ & 1.535 & 6.421 & 100 & 8,925 & 75,069 \\
\hline \multicolumn{8}{|l|}{ Out of Sample } \\
\hline Op T2M & 0.295 & 0.303 & 1.669 & 5.235 & 0.027 & 0.164 & 1.479 \\
\hline Op Moneyness & 0.972 & 0.047 & -0.422 & 2.614 & 0.850 & 0.977 & 1.060 \\
\hline Op Price/Vega & 0.189 & 0.642 & 82.578 & 9,353 & 0.013 & 0.109 & 90.927 \\
\hline Op Trading vol & 811 & 1,977 & 20.398 & 1,232 & 1 & 178 & 157,542 \\
\hline Op Open int & 12,856 & 16,113 & 3.421 & 22.919 & 100 & 7,722 & 214,048 \\
\hline
\end{tabular}


Table 2: Parameter Estimate

This table reports parameter estimates and standard deviations for three nested factor models. The models are normalized by setting $\nu_{k}=1$, for $k=1, \cdots, K$. The one-, two- and three-factor models derived in the paper are estimated using the daily data of S\&P 500 Index, two index futures and three index options described in Section 5. The loglikelihood for each model and test statistics $\xi_{\mathbf{w}}$ computed in equation (4.5) is presented at the end of the table.

\begin{tabular}{|c|c|c|c|c|c|c|}
\hline \multirow[t]{2}{*}{ Parameter } & \multicolumn{2}{|c|}{ One Factor } & \multicolumn{2}{|c|}{ Two Factors } & \multicolumn{2}{|c|}{ Three Factors } \\
\hline & Estimate & Std.Err. & Estimate & Std.Err. & Estimate & Std.Err. \\
\hline$\mu_{0}$ & 0.014156 & 0.062649 & 0.001751 & 0.490664 & 0.020641 & 0.000306 \\
\hline$\mu_{1}$ & -0.019612 & 1.202200 & -0.120038 & 3.543429 & 0.326886 & 0.001813 \\
\hline$\mu_{2}$ & - & - & 0.501187 & 0.000786 & 0.241127 & 0.011074 \\
\hline$\mu_{3}$ & - & - & - & - & 0.238038 & 0.000913 \\
\hline$\gamma_{0}$ & 0.005084 & 0.021024 & 0.005787 & 0.060267 & 0.005612 & $7.60 \mathrm{e}-05$ \\
\hline$\gamma_{1}$ & 0.091091 & 0.226156 & 0.115763 & 0.453376 & 0.006901 & 0.000180 \\
\hline$\gamma_{2}$ & - & - & $1.72 \mathrm{e}-05$ & $1.21 \mathrm{e}-07$ & 0.003152 & $5.24 \mathrm{e}-05$ \\
\hline$\gamma_{3}$ & - & - & - & - & 0.067161 & 0.004048 \\
\hline$\xi_{1}$ & 3.364535 & 6.302816 & 2.667334 & 11.54964 & 2.230514 & 0.006613 \\
\hline$\xi_{2}$ & - & - & 3.409025 & 0.474828 & 2.230497 & 0.032053 \\
\hline$\xi_{3}$ & - & - & - & - & 1.719731 & 0.079500 \\
\hline$\zeta_{1}$ & 0.103510 & 0.442547 & 0.052931 & 0.450232 & 0.093168 & 0.000992 \\
\hline$\zeta_{2}$ & - & - & 0.078183 & 0.000923 & 0.013873 & 0.000221 \\
\hline$\zeta_{3}$ & - & - & - & - & 0.084855 & 0.002533 \\
\hline$\alpha_{0}$ & 0.279563 & 0.710415 & 0.351444 & 0.043135 & 0.376432 & 0.017518 \\
\hline$\alpha_{1}$ & 0.115896 & 3.850134 & 0.356801 & 6.467518 & 1.500325 & 0.013313 \\
\hline$\alpha_{2}$ & - & - & -0.476146 & 0.362013 & 1.181081 & 0.008354 \\
\hline
\end{tabular}

(to be continued on next page...) 
Table 2: (continued)

\begin{tabular}{|c|c|c|c|c|c|c|}
\hline \multirow[t]{2}{*}{ Parameter } & \multicolumn{2}{|c|}{ One Factor } & \multicolumn{2}{|c|}{ Two Factors } & \multicolumn{2}{|c|}{ Three Factors } \\
\hline & Estimate & Std.Err. & Estimate & Std.Err. & Estimate & Std.Err. \\
\hline$\alpha_{3}$ & - & - & - & - & 0.059578 & 0.002488 \\
\hline$\beta$ & -0.421196 & 12.37646 & -1.860492 & 0.960758 & -5.662848 & 0.415460 \\
\hline$\sigma_{F u 1}$ & 0.022757 & 0.001289 & 0.009532 & 0.003030 & 0.009446 & 0.000655 \\
\hline$\sigma_{F u 2}$ & 0.017257 & 0.001578 & 0.000290 & 0.065585 & 0.000790 & $3.36 \mathrm{e}-05$ \\
\hline$\sigma_{O p 1}$ & 0.012955 & 0.006663 & 0.015889 & 0.025387 & 0.016573 & 0.000553 \\
\hline$\sigma_{O p 2}$ & 0.010243 & 0.008514 & 0.009056 & 0.066390 & 0.010097 & 0.000281 \\
\hline$\sigma_{O p 3}$ & 0.013427 & 0.017725 & 0.010930 & 0.009359 & 0.008439 & 0.000746 \\
\hline Loglikelihood & \multicolumn{2}{|c|}{$25,525.29$} & \multicolumn{2}{|c|}{$28,491.26$} & \multicolumn{2}{|c|}{$28,853.48$} \\
\hline$\xi_{\mathrm{w}}$ & \multicolumn{2}{|c|}{845.9943} & \multicolumn{2}{|c|}{997.2979} & \multicolumn{2}{|c|}{$18,231.71$} \\
\hline$\chi_{99 \%}^{2}(K+1)$ & \multicolumn{2}{|c|}{9.21} & \multicolumn{2}{|c|}{11.35} & \multicolumn{2}{|c|}{13.28} \\
\hline
\end{tabular}


Table 3: In-Sample Absolute Percentage Pricing (Forecasting) Errors This table reports the in-sample pricing errors, which are measured as the absolute difference between model implied price and the observed price as a percentage of the observed price. For the index which is not tradable, the interpretation is in terms of forecasting error. For the index, we report on the log index value, while for the futures and options, the actual prices are examined. Fu1 refers to the short-term futures, and Fu2 refers to the medium-term futures. Op1 represents at-the-money options with short maturity (ATMSM). Op2 stands for out-of-the-money options with medium maturity (OTM-MM), and Op3 are out-of-the-money options with long maturity (OTM-LM). There are 1,506 days in the sample.

\begin{tabular}{ccccccccc}
\hline \multirow{2}{*}{ Securities } & \multicolumn{2}{c}{ One Factor } & & \multicolumn{2}{c}{ Two Factors } & & \multicolumn{2}{c}{ Three Factors } \\
\cline { 2 - 3 } & Mean & Std.Dev. & & Mean & Std.Dev. & & Mean & Std.Dev. \\
\hline Fu1 & 0.001108 & 0.001086 & & 0.001109 & 0.001088 & & 0.001112 & 0.001095 \\
Fu2 & 0.005233 & 0.003227 & & $4.86 \mathrm{e}-06$ & $4.70 \mathrm{e}-06$ & & $3.98 \mathrm{e}-05$ & $4.12 \mathrm{e}-05$ \\
Op1 & 0.089782 & 0.164550 & & 0.105236 & 0.178787 & & 0.107083 & 0.179544 \\
Op2 & 0.097314 & 0.152559 & & 0.082150 & 0.113209 & & 0.095510 & 0.128891 \\
Op3 & 0.104998 & 0.142086 & & 0.087099 & 0.109224 & & 0.067479 & 0.084363 \\
\hline
\end{tabular}


Table 4: Out-of-the-Sample Absolute Percentage Options Pricing Errors This table reports the out-of-the-sample options pricing errors, which are measured as the absolute difference between model implied price and the observed price as a percentage of the observed price. There are 1,506 days and 72,706 out-of-the-sample observations in total. The options are divided into 15 groups based on the maturity and moneyness. The mean of absolute percentage options pricing errors for each group is reported.

\begin{tabular}{llrrrrr}
\hline \multirow{2}{*}{ Maturity } & Model & \multicolumn{5}{c}{ Moneyness(I/K) } \\
\cline { 3 - 7 }$<60$ days & one-factor & 0.545435 & 0.466165 & 0.154574 & 0.048615 & 0.028152 \\
& two-factor & 0.529769 & 0.466957 & 0.165987 & 0.044368 & 0.025586 \\
& three-factor & 0.519731 & 0.436678 & 0.163203 & 0.045412 & 0.028491 \\
\multirow{2}{*}{$60-180$} & & & & & \\
& one-factor & 0.360111 & 0.122878 & 0.061926 & 0.053554 & 0.033464 \\
& two-factor & 0.329843 & 0.111317 & 0.057041 & 0.046736 & 0.025826 \\
& three-factor & 0.336643 & 0.106182 & 0.057058 & 0.049048 & 0.033831 \\
& & & & & & \\
\hline \multirow{2}{*}{$>180$} & one-factor & 0.158253 & 0.076913 & 0.072129 & 0.075685 & 0.087410 \\
& two-factor & 0.127078 & 0.063742 & 0.054993 & 0.058140 & 0.080321 \\
\hline & three-factor & 0.109486 & 0.056811 & 0.051816 & 0.054455 & 0.068869 \\
\hline
\end{tabular}




\section{References}

[1] Ball, C., and A., Roma (1994), "Stochastic Volatility Option Pricing", Journal of Financial and Quantitative Analysis, 29, 589-607.

[2] Black, F., and M., Scholes (1973), "The Pricing of Options and Corporate Liabilities", Journal of Political Economy, 81, 637-659.

[3] Brennan, M., and E., Schwartz (1990), "Arbitrage in Stock Index Futures", Journal of Business, Vol. 63, 7-31.

[4] Chen, R., and L., Scott (1993), "Maximum Likelihood Estimation of a Multi-Factor Equilibrium Model of the Term Structure of Interest Rates", Journal of Fixed Income, December, 1993, 14-31.

[5] Constantinides, G. (1992), "A Theory of the Nominal Term Structure of Interest Rates", Review of Financial Studies, 5, 531-552.

[6] Cox, J., Ingersoll, J., and S., Ross (1981), "The Relation between Forward Prices and Future Prices", Journal of Financial Economics, 9, 321-346.

[7] Cox, J., Ingersoll, J., and S., Ross (1985a), "An Intertemporal General Equilibrium Model of Asset Prices", Econometrica, 53, 363-384.

[8] Cox, J., Ingersoll, J., and S., Ross (1985b), "A Theory of the Term Structure of Interest Rates", Econometrica, 53, 385-407.

[9] Dai, Q., and K., Singleton (2000), "Specification Analysis of Affine Term Structure Models", Journal of Finance, Vol 55, 1943-1978.

[10] Dai, Q., and K., Singleton (2003), "Term Structure Dynamics in Theory and Reality", The Review of Financial Studies, 16, 631-678.

[11] Duffie, D., and R., Kan (1996), "A Yield-Factor Model of Interest Rates", Mathematical Finance, 6, 379-406. 
[12] Duffie, D., Pan, J., and K., Singleton (2000), "Transform Analysis and Asset Pricing for Affine Jump-Diffusions", Econometrica, 68, 1343-1376.

[13] French, K. R. (1983), "A Comparison of Futures and Forward Prices", Journal of Financial Economics, Vol. 12, 311-342.

[14] Gourieroux, C., Monfort, A., and A., Trognon (1984), Pseudo-maximum Likelihood Methods: Theory, Econometrica, 42, 681-700.

[15] Harrison, J., and D., Kreps (1979), "Martingales and Arbitrage in Multiperiod Securities Markets", Journal of Economic Theory, 20, 381-408.

[16] Heston, S.(1993) "A Closed-Form Solution for Options with Stochastic Volatility with Applications to Bond and Currency Options", The Review of Financial Studies, $6,327-343$.

[17] Li, J.(2012) "Option-Implied Volatility Factors and the Cross-Section of Market Risk Premia", Journal of Banking and Finance, Jan 2012, Vol 36, Issue 1, p. 249-260 .

[18] Merton, R. (1973), "The Theory of Rational Option Pricing", Bell Journal of Economics and Management Science, 4, 141-183.

[19] Roll, R. (1977), “A critique of the asset pricing theory's tests Part I: On past and potential testability of the theory", Journal of Financial Economics, 4 (2), 129176.

[20] Schwartz, E. (1997), "The Stochastic Behavior of Commodity Prices: Implications for Valuation and Hedging", Journal of Finance, vol 52, 923-973.

[21] Wan, E. A., and R., Van Der Merwe (2000), "The Unscented Kalman Filter for Nonlinear Estimation", Adaptive Systems for Signal Processing, Communications, and Control Symposium 2000. AS-SPCC. The IEEE 2000, 153-158.

[22] White, H. (1982), "Maximum Likelihood Estimation of Misspecified Models", Econometrica, 50, 1-25. 
[23] Xu, P. (2006), "Is S\&P 500 Index tradable?", Manuscript.

[24] Xu, P. (2007), "S\&P 500 Index Options and Put-Call Parity", Manuscript. 